International Mathematical Forum, 2, 2007, no. 14, 633 - 674

\title{
Conjugate Journey in Optimal Control
}

\author{
Javier F Rosenblueth \\ IIMAS-UNAM, Apartado Postal 20-726 \\ México DF 01000, México \\ jfrl@servidor.unam.mx
}

\begin{abstract}
This paper seeks to unify, and improve, different approaches to conjugacy applicable to certain classes of optimal control problems. This covers the classical notion of conjugate points in the calculus of variations, various extensions of this concept to optimal control, and the recently developed theory of conjugate intervals.
\end{abstract}

Keywords: Calculus of variations, optimal control, conjugate points, conjugate intervals, second order necessary conditions, normality

Mathematics Subject Classification: 49K15

Jarndyce and Jarndyce drones on. This scarecrow of a suit has, in course of time, become so complicated that no man alive knows what it means. The parties to it understand it least; but it has been observed that no two Chancery lawyers can talk about it for five minutes without coming to a total disagreement as to all the premises.

Charles Dickens, Bleak House

\section{Introduction}

The theory of conjugate points in optimal control (the cause in hand) is not precisely Jarndyce and Jarndyce. But there is an undeniable similarity to it. Most readers will probably agree that the main objective of this theory is to generalize the conditions of Jacobi from calculus of variations to an optimal control setting. But both the word "generalize," and the notion of "conjugate points" in the theory of Jacobi, may have entirely different meanings. In fact, it has been observed that some authors (sometimes even the same author in different papers) may come, in less than five minutes, to a total disagreement as to some of the premises. 
Let us briefly explain possible causes of this situation. Consider first the classical necessary condition of Jacobi. In most texts treating the simple fixed-endpoint problem in the calculus of variations, this condition states that "there are no conjugate points in the underlying open time interval." In the references, we have included the titles of eleven well-known books ([1], [6], $[9],[10],[11],[12],[14],[15],[18],[22],[26])$ where this condition is indeed established as necessary. However, not two of these texts agree totally on all the premises, namely, the space where the function to be minimized is defined, the assumptions on the sets and functions delimiting the problem and, even more, the definition itself of conjugate points. All claim that their theorem corresponds to "the" condition of Jacobi. Thus, someone coming across these books (and not only for the first time) may very easily get confused. As Ewing [10] says, "A reader meeting the calculus of variations for the first time is likely to feel that a great many things are not completely clear. This is normal. To feel otherwise is either a mark of genius or indication of real trouble."

In this paper we shall concentrate on this necessary condition, and we intend to give a clear meaning to it. Therefore, our first objective will be to unify the different approaches that can be found at least in those references. Once this is done, we shall proceed to "generalize" this point of view to optimal control. Now, this condition is necessary because it holds (under certain assumptions) for trajectories satisfying a well-known second order necessary condition. Thus, for optimal control problems, a corresponding second order condition might lead us to possible definitions of conjugacy. But, once again, this aspect of the theory varies dramatically between different authors. In [30], for example, it is claimed in an "important remark" that the case when inequality constraints are present is within the scope of the paper, where only the equality constraint case is studied. According to [17], "this is neither clear nor convincing," and the presence of this kind of constraints becomes a crucial feature in the second order conditions obtained. There, it is fundamental for the control set to be convex but, in [29], this assumption is no longer required.

We could give more examples of unclear claims, but this is not our objective. We are mainly interested in finding an "appropriate" notion of conjugate points for certain optimal problems and so, to avoid any possible misunderstandings, we shall deal with the fixed-endpoint control problem of Lagrange (without isoperimetric constraints), as presented in [14], where second order conditions are clearly established. Also, this choice will allow us to keep notational complexity to a minimum. It is important to mention, nevertheless, that the different approaches to conjugacy presented in this paper can be generalized to more complex problems. The theory we develop certainly depends on the second order conditions, but not the approaches themselves.

Let us now consider the question of how to generalize the notion of conjugacy to optimal control (in particular, to our problem of interest). This 
question is perhaps the main cause of disagreement. In [5] and [8] one can find some of the first attempts in this direction but, in [32], it is assured repeatedly that both papers are incorrect (this claim is denied by Bernhard in a reply following [32]). In subsequent papers such as [28], [30], [31] and [33], one encounters different definitions of conjugate points which are not all equivalent even if the problems considered are reduced to the same fixedendpoint problem. In the calculus of variations context, however, all these notions coincide, and in this sense all generalize the classical theory. However, in [17] and [29], this is no longer the case. There, the sets of conjugate points, in the calculus of variations setting, contain (but in general do not coincide with) that of classical conjugate points in the underlying time open interval under nonsingularity assumptions. One of our main objectives will be to unify also this rather controversial aspect of the theory.

This paper can be seen as a journey through different approaches to conjugacy. Though we cannot cover all possible attempts to generalize this theory to optimal control, we have chosen one line of research that we consider to be quite representative. We shall begin, for the calculus of variations problem, with a summary of the main aspects of the theory according to Hestenes. Our second stop corresponds to an "improvement" (in the sense that the basic assumptions are weakened) of this classical (linear system) approach yielding the necessary condition of Jacobi. We also present, and improve as before, a second (continuity) approach found in the literature for which the definition of conjugate points differs from the previous one. We then turn to the fixedendpoint control problem based, once more, on Hestenes, and study various definitions of conjugate points found in different papers which correspond to a generalization of the linear system approach. We improve these definitions and also introduce an extension of the continuity approach.

Finally, we arrive to the point of view first introduced in [17], and modified in [29] for the normal case. Let us briefly mention that, in both cases, the nonemptiness of the sets defined has been established merely as a sufficient condition for the existence of negative second variations. Also (we hope most readers will agree) the main idea of characterizing a condition is, generally, to obtain a simpler way of verifying it. However, even in the calculus of variations, simple examples show that to solve the question of nonemptiness of these sets may be much more difficult than checking if that condition holds. We introduce new sets containing those of [17] and [29] for which these two features are solved, as well as an extension of the linear system and continuity approaches as generalized for the optimal control problem. Several examples illustrate the usefulness of the new sets introduced throughout the journey.

\section{Calculus of variations: a classical approach}


In this section we shall deal with the simple fixed-endpoint problem in the calculus of variations. We state the problem and summarize well-known sets of necessary and sufficient conditions for optimality. Those conditions expressed in terms of the first and second variations will then be characterized by means of Euler's equation and Jacobi's theory. The approach we follow is essentially that of Hestenes [14].

Suppose we are given an interval $T:=\left[t_{0}, t_{1}\right]$ in $\mathbf{R}$, two points $\xi_{0}, \xi_{1}$ in $\mathbf{R}^{n}$, a relatively open set $\mathcal{A}$ of $T \times \mathbf{R}^{n} \times \mathbf{R}^{n}$, and a function $L$ mapping $T \times \mathbf{R}^{n} \times \mathbf{R}^{n}$ to $\mathbf{R}$. Let $X$ be the space of all piecewise $C^{1}$ functions mapping $T$ to $\mathbf{R}^{n}$, set

$$
\begin{aligned}
& X(\mathcal{A}):=\{x \in X \mid(t, x(t), \dot{x}(t)) \in \mathcal{A}(t \in T)\}, \\
& X_{e}(\mathcal{A}):=\left\{x \in X(\mathcal{A}) \mid x\left(t_{0}\right)=\xi_{0}, x\left(t_{1}\right)=\xi_{1}\right\}
\end{aligned}
$$

and consider the functional $I: X \rightarrow \mathbf{R}$ given by $I(x):=\int_{t_{0}}^{t_{1}} L(t, x(t), \dot{x}(t)) d t$ $(x \in X)$. The problem we shall deal with, which we label $\mathrm{P}(\mathcal{A})$, is that of minimizing $I$ over $X_{e}(\mathcal{A})$.

Elements of $X$ will be called trajectories, and a trajectory $x$ solves $\mathrm{P}(\mathcal{A})$ if $x \in X_{e}(\mathcal{A})$ and $I(x) \leq I(y)$ for all $y \in X_{e}(\mathcal{A})$. For local minima, a trajectory $x$ will be called a strong or a weak minimum of $\mathrm{P}(\mathcal{A})$ if, for some $\epsilon>0, x$ solves $\mathrm{P}\left(T_{0}(x ; \epsilon) \cap \mathcal{A}\right)$ or $\mathrm{P}\left(T_{1}(x ; \epsilon) \cap \mathcal{A}\right)$ respectively where, for all $x \in X$ and $\epsilon>0$,

$$
\begin{gathered}
T_{0}(x ; \epsilon):=\left\{(t, y, v) \in T \times \mathbf{R}^{n} \times \mathbf{R}^{n}:|x(t)-y|<\epsilon\right\}, \\
T_{1}(x ; \epsilon):=\left\{(t, y, v) \in T_{0}(x ; \epsilon):|\dot{x}(t)-v|<\epsilon\right\} .
\end{gathered}
$$

For any $x \in X$ we shall use the notation $(\tilde{x}(t))$ to represent $(t, x(t), \dot{x}(t))$. For $u$ equal to $x, \dot{x}$ or both, we shall say that $L \in C^{r}(\mathcal{A} ; u)(r \geq 1)$ if $L$ is continuous on $\mathcal{A}$ and $C^{r}(\mathcal{A})$ with respect to $u$.

For all $x \in X$ consider the first variation of $I$ along $x$ given by

$$
I^{\prime}(x ; y):=\int_{t_{0}}^{t_{1}}\left\{\left\langle L_{x}(\tilde{x}(t)), y(t)\right\rangle+\left\langle L_{\dot{x}}(\tilde{x}(t)), \dot{y}(t)\right\rangle\right\} d t \quad(y \in X)
$$

and the second variation of $I$ along $x$ given by

$$
I^{\prime \prime}(x ; y):=\int_{t_{0}}^{t_{1}} 2 \Omega(t, y(t), \dot{y}(t)) d t \quad(y \in X)
$$

where, for all $(t, y, \dot{y}) \in T \times \mathbf{R}^{n} \times \mathbf{R}^{n}$,

$$
2 \Omega(t, y, \dot{y}):=\left\langle y, L_{x x}(\tilde{x}(t)) y\right\rangle+2\left\langle y, L_{x \dot{x}}(\tilde{x}(t)) \dot{y}\right\rangle+\left\langle\dot{y}, L_{\dot{x} \dot{x}}(\tilde{x}(t)) \dot{y}\right\rangle .
$$

Define the set of admissible variations by $Y:=\left\{y \in X \mid y\left(t_{0}\right)=y\left(t_{1}\right)=0\right\}$ and consider the following sets:

$$
\mathcal{E}:=\left\{x \in X \mid I^{\prime}(x ; y)=0 \text { for all } y \in Y\right\}
$$




$$
\begin{gathered}
\mathcal{H}:=\left\{x \in X \mid I^{\prime \prime}(x ; y) \geq 0 \text { for all } y \in Y\right\}, \\
\mathcal{L}:=\left\{x \in X \mid L_{\dot{x} \dot{x}}(\tilde{x}(t)) \geq 0 \text { for all } t \in T\right\}, \\
\mathcal{W}(\mathcal{A}):=\{x \in X(\mathcal{A}) \mid E(t, x(t), \dot{x}(t), u) \geq 0 \\
\text { for all } \left.(t, u) \in T \times \mathbf{R}^{n} \text { with }(t, x(t), u) \in \mathcal{A}\right\}
\end{gathered}
$$

where the Weierstrass "excess function" $E: T \times \mathbf{R}^{3 n} \rightarrow \mathbf{R}$ is given by

$$
E(t, x, \dot{x}, u):=L(t, x, u)-L(t, x, \dot{x})-\left\langle u-\dot{x}, L_{\dot{x}}(t, x, \dot{x})\right\rangle .
$$

Elements of $\mathcal{E} \cap C^{1}$ are called extremals, elements of $\mathcal{L}$ are said to satisfy the condition of Legendre, and elements of $\mathcal{W}(\mathcal{A})$ to satisfy the condition of Weierstrass.

The following theorem gives necessary conditions for a solution of $\mathrm{P}(\mathcal{A})$ where $\mathcal{A}$ is any relatively open set of $T \times \mathbf{R}^{n} \times \mathbf{R}^{n}$. In particular, under the assumptions of the theorem, if $x$ is a weak minimum of $\mathrm{P}(\mathcal{A})$ then $x$ belongs to $\mathcal{E}, \mathcal{H}, \mathcal{L}$ and $\mathcal{W}\left(T_{1}(x ; \epsilon) \cap \mathcal{A}\right)$ for some $\epsilon>0$. On the other hand, if $x$ is a strong minimum of $\mathrm{P}(\mathcal{A})$ then $x$ belongs to $\mathcal{E}, \mathcal{H}, \mathcal{L}$ and $\mathcal{W}(\mathcal{A})$ since, for any $\epsilon>0, x \in \mathcal{W}\left(T_{0}(x ; \epsilon) \cap \mathcal{A}\right) \Leftrightarrow x \in \mathcal{W}(\mathcal{A})$.

2.1 Theorem: If $x$ solves $\mathrm{P}(\mathcal{A})$ then $x$ belongs to $\mathcal{E}, \mathcal{H}, \mathcal{L}$ and $\mathcal{W}(\mathcal{A})$ whenever $L$ and its derivatives involved are continuous on $\mathcal{A}$.

Consider now the following sets obtained by slightly strengthening those defined for necessary conditions:

$$
\begin{gathered}
\mathcal{H}^{\prime}:=\left\{x \in X \mid I^{\prime \prime}(x ; y)>0 \text { for all } y \in Y, y \neq 0\right\}, \\
\mathcal{L}^{\prime}:=\left\{x \in X \mid L_{\dot{x} \dot{x}}(\tilde{x}(t))>0 \text { for all } t \in T\right\}, \\
\mathcal{W}(\mathcal{A}, \epsilon):=\left\{x_{0} \in X(\mathcal{A}) \mid E(t, x, \dot{x}, u) \geq 0 \text { for all }(t, x, \dot{x}, u) \in T \times \mathbf{R}^{3 n}\right. \text { with } \\
\left.(t, x, \dot{x}) \in T_{1}\left(x_{0} ; \epsilon\right) \text { and }(t, x, u) \in \mathcal{A}\right\} .
\end{gathered}
$$

The following theorem gives sufficient conditions for local minima. In [14] it is proved directly without referring to Mayer fields, Hamilton-Jacobi theory, Riccati equations or conjugate points.

2.2 Theorem: Suppose $L \in C^{2}(\mathcal{A} ; x, \dot{x})$ and $x \in X_{e}(\mathcal{A}) \cap C^{1}$. Then:

a. $x \in \mathcal{E} \cap \mathcal{H}^{\prime} \cap \mathcal{L}^{\prime} \Rightarrow x$ is a strict weak minimum of $\mathrm{P}(\mathcal{A})$.

b. $x \in \mathcal{E} \cap \mathcal{H}^{\prime} \cap \mathcal{L}^{\prime} \cap \mathcal{W}(\mathcal{A} ; \epsilon)$ for some $\epsilon>0 \Rightarrow x$ is a strict strong minimum of $\mathrm{P}(\mathcal{A})$.

Now, the sets $\mathcal{E}, \mathcal{H}$ and $\mathcal{H}^{\prime}$, as defined above, are expressed in terms of variations of $I$. The remaining of this section will be devoted to some wellknown characterizations of these sets. 
2.3 Proposition: Suppose $L \in C^{1}(\mathcal{A} ; x, \dot{x})$ and $x \in X(\mathcal{A})$. Then $x \in \mathcal{E}$ $\Leftrightarrow$ there exists $c \in \mathbf{R}^{n}$ such that

$$
L_{\dot{x}}(\tilde{x}(t))=\int_{t_{0}}^{t} L_{x}(\tilde{x}(s)) d s+c \quad(t \in T) .
$$

2.4 Remark: The above equation is the integral form of Euler's equation

$$
\frac{d}{d t} L_{\dot{x}}(\tilde{x}(t))=L_{x}(\tilde{x}(t)) \quad(t \in T) .
$$

If $\dot{x}$ has a discontinuity, the derivative $d / d t$ is to be interpreted as a left- or a right-hand derivative, and it holds even if $x$ fails to have a second derivative. It is important, in the theory to follow, to bear in mind that (under the assumptions of 2.3) if $x \in \mathcal{E}$ then $x$ satisfies Euler's equation, but the converse may not hold. This is easily illustrated by, for example, setting $L(t, x, \dot{x})=$ $\left(\dot{x}^{2}-x^{2}\right) / 2, T=[0,2 \pi], x_{0}(t):=\sin t$ if $t \in[0, \pi]$, and $x_{0}(t):=0$ if $t \in[\pi, 2 \pi]$. Then $x_{0}$ satisfies Euler's equation, but $x_{0} \notin \mathcal{E}$ since there is no $c \in \mathbf{R}$ satisfying $\dot{x}_{0}(t)=\int_{0}^{t}-x_{0}(s) d s+c$ for all $t \in T$. For the converse note that, if $x$ satisfies Euler's equation, then $x \in \mathcal{E}$ if the function $t \mapsto L_{\dot{x}}(\tilde{x}(t))(t \in T)$ belongs to $X$.

We shall find convenient to restate the characterization given in 2.3 as follows ( ${ }^{*}$ ) denotes transpose).

2.5 Note: For all $(t, x, u, p) \in T \times \mathbf{R}^{3 n}$ let $H(t, x, u, p):=\langle p, u\rangle-$ $L(t, x, u)$, and set

$M(x):=\left\{p \in X \mid \dot{p}(t)=-H_{x}^{*}(\tilde{x}(t), p(t))\right.$ and $\left.H_{u}(\tilde{x}(t), p(t))=0(t \in T)\right\}(x \in X)$.

Then (under the assumptions of 2.3) $x \in \mathcal{E} \Leftrightarrow M(x) \neq \emptyset$.

For the sets $\mathcal{H}$ and $\mathcal{H}^{\prime}$ we invoke the theory of conjugate points. We shall later see other approaches but, for the time being, let us continue with that of Hestenes.

2.6 Definition: Given $x \in X$, a point $s \in\left(t_{0}, t_{1}\right]$ is called conjugate to $t_{0}$ on $x$ if there exists $y \in X$ with $y\left(t_{0}\right)=y(s)=0, y \not \equiv 0$ on $\left(t_{0}, s\right)$, and $y$ satisfies Jacobi's equation

$$
\frac{d}{d t}\left[L_{\dot{x} x}(\tilde{x}(t)) y(t)+L_{\dot{x} \dot{x}}(\tilde{x}(t)) \dot{y}(t)\right]=L_{x x}(\tilde{x}(t)) y(t)+L_{x \dot{x}}(\tilde{x}(t)) \dot{y}(t) \quad(t \in T) .
$$

We denote by $\mathcal{C}(x)$ the set of points conjugate to $t_{0}$ on $x$.

Consider the set $\mathcal{J}:=\left\{x \in X \mid \mathcal{C}(x) \cap\left(t_{0}, t_{1}\right)=\emptyset\right\}$ whose elements are said to satisfy the condition of Jacobi. This set does not characterize $\mathcal{H}$ 
in general since we may very well have points belonging to $\left(t_{0}, t_{1}\right)$, which are conjugate to $t_{0}$ on $x$, but $x \in \mathcal{H}$. To illustrate this fact suppose, for example, that $L(t, x, \dot{x})=x \dot{x}$ for all $(t, x, \dot{x}) \in T \times \mathbf{R} \times \mathbf{R}$. Then, for any $x \in X$, all points belonging to $\left(t_{0}, t_{1}\right]$ are conjugate to $t_{0}$ on $x$ (thus $x \notin \mathcal{J}$ ), but $x \in \mathcal{H}$ since $I^{\prime \prime}(x ; y)=0$ for all $y \in Y$. For $\mathcal{H}$ and $\mathcal{J}$ to be equivalent, one requires some additional assumptions on $x$. In [14], the crucial assumption on the minimizing trajectory $x$ is the strengthened condition of Legendre.

2.7 Theorem: Suppose $L \in C^{2}(\mathcal{A})$ and $x \in X(\mathcal{A}) \cap \mathcal{L}^{\prime} \cap C^{1}$ solves $\mathrm{P}(\mathcal{A})$. Then $x \in \mathcal{J}$.

This is a consequence of Theorem 2.1 and the following result (see [14, p 124].

2.8 Theorem: Suppose $L \in C^{2}(\mathcal{A})$ and $x \in X(\mathcal{A})$ is an extremal in $\mathcal{L}^{\prime}$. Then $x \in \mathcal{H} \Rightarrow x \in \mathcal{J}$.

In [14, p 134], the set $\mathcal{H}$ is actually characterized in terms of conjugate points. To state this result, let us call a trajectory $x$ nonsingular if $\left|L_{\dot{x} \dot{x}}(\tilde{x}(t))\right| \neq 0$ for all $t \in T$. Note that $x \in \mathcal{L}^{\prime} \Leftrightarrow x \in \mathcal{L}$ is nonsingular.

2.9 Theorem: Suppose $L \in C^{2}(\mathcal{A})$ and $x \in X(\mathcal{A})$ is a nonsingular extremal. Then $x \in \mathcal{H} \Leftrightarrow x \in \mathcal{L} \cap \mathcal{J}$.

For sufficiency, consider the set $\mathcal{J}^{\prime}:=\{x \in X \mid \mathcal{C}(x)=\emptyset\}$. Analogous to the above result, the set $\mathcal{H}^{\prime}$ can be characterized in terms of $\mathcal{L}$ and $\mathcal{J}^{\prime}$ (see $[14, \mathrm{p} 134])$.

2.10 Theorem: Suppose $L \in C^{2}(\mathcal{A})$ and $x \in X(\mathcal{A})$ is a nonsingular extremal. Then $x \in \mathcal{H}^{\prime} \Leftrightarrow x \in \mathcal{L} \cap \mathcal{J}^{\prime}$.

\section{Calculus of variations: weaker assumptions}

In the classical literature in the calculus of variations, one can find at least two different approaches to conjugacy related to second order necessary conditions. Both state that, under certain assumptions, if $x$ solves $\mathrm{P}(\mathcal{A})$ then $x$ satisfies Jacobi's necessary condition, that is, the open interval $\left(t_{0}, t_{1}\right)$ contains no conjugate points to $t_{0}$ on $x$.

One of them is somehow represented by Hestenes and it requires $x$ to be nonsingular. For reasons that will become apparent presently, we shall call this the "linear system" approach. Though this is perhaps the most common one, the literature includes a different approach for which the assumption of nonsingularity of the extremal is not imposed. We shall call it the "continuity" approach. As we shall see in this section, the two approaches include some assumptions on both the Lagrangian and the trajectory which can be weakened 
considerably.

\section{The linear system approach}

Let us begin with the first approach which yields Theorem 2.8. The proof of this result, given in [14], is based on the following observations.

- For any $x \in X$ consider the (so-called "accessory") problem, which we label (AP), of minimizing

$$
K(y):=\int_{t_{0}}^{t_{1}} \Omega(t, y(t), \dot{y}(t)) d t
$$

subject to $y \in X$ and $y\left(t_{0}\right)=y\left(t_{1}\right)=0$, that is, minimize $I^{\prime \prime}(x ; \cdot) / 2$ over $Y$.

- For any $x \in X$ let $\mathcal{E}(x)$ be the set of trajectories $y \in X$ for which there exists $c \in \mathbf{R}^{n}$ such that

$$
\Omega_{\dot{y}}(\tilde{y}(t))=\int_{t_{0}}^{t} \Omega_{y}(\tilde{y}(s)) d s+c \quad(t \in T) .
$$

It is well-known that, if $L \in C^{2}(\mathcal{A} ; x, \dot{x})$ and $x \in X(\mathcal{A}) \cap \mathcal{L}^{\prime} \cap C^{1}$, then $\mathcal{E}(x) \subset C^{1}$ (see [14, p 123). Note that, in terms of 2.5, if we let

$$
\tilde{H}(t, y, v, q):=\langle q, v\rangle-\Omega(t, y, v) \quad\left((t, y, v, q) \in T \times \mathbf{R}^{3 n}\right)
$$

and define $\tilde{M}(y)$ as the set of trajectories $q \in X$ satisfying

$$
\dot{q}(t)=-\tilde{H}_{y}^{*}(\tilde{y}(t), q(t))\left[=\Omega_{y}^{*}(\tilde{y}(t))\right], \quad 0=\tilde{H}_{v}^{*}(\tilde{y}(t), q(t))\left[=q(t)-\Omega_{\dot{y}}^{*}(\tilde{y}(t))\right]
$$

then $y \in \mathcal{E}(x) \Leftrightarrow \tilde{M}(y) \neq \emptyset$.

- For any $x \in X$ let $\mathcal{J}(x)$ be the set of trajectories $y \in X$ that satisfy Euler's equation with respect to the integrand $\Omega$, that is,

$$
\frac{d}{d t} \Omega_{\dot{y}}(\tilde{y}(t))=\Omega_{y}(\tilde{y}(t)) \quad(t \in T) .
$$

Since

$\Omega_{y}^{*}(t, y, \dot{y})=L_{x x}(\tilde{x}(t)) y+L_{x \dot{x}}(\tilde{x}(t)) \dot{y}, \quad \Omega_{\dot{y}}^{*}(t, y, \dot{y})=L_{\dot{x} x}(\tilde{x}(t)) y+L_{\dot{x} \dot{x}}(\tilde{x}(t)) \dot{y}$,

$y \in \mathcal{J}(x)$ if and only if $y$ satisfes Jacobi's equation. For any $x \in X$ we have $\mathcal{E}(x) \subset \mathcal{J}(x)$. Also, if $y \in \mathcal{J}(x)$ is such that the function $t \mapsto \Omega_{\dot{y}}(\tilde{y}(t))(t \in T)$ belongs to $X$, then $y \in \mathcal{E}(x)$.

We shall also make use of the next result which follows by a direct substitution. 
3.1 Proposition: Suppose $x \in \mathcal{L}^{\prime}$ and $(y, q) \in X \times X$. Then the following are equivalent:

a. $q \in \tilde{M}(y)$.

b. $(y, q)$ satisfies, for all $t \in T$, the linear system

$$
\dot{y}(t)=A(t) y(t)+B(t) q(t), \quad \dot{q}(t)=C(t) y(t)-A^{*}(t) q(t) \quad(t \in T)
$$

which we label $(\mathrm{J})$, where

$$
\begin{gathered}
A(t)=-L_{\dot{x} \dot{x}}^{-1}(\tilde{x}(t)) L_{\dot{x} x}(\tilde{x}(t)) \\
B(t)=L_{\dot{x} \dot{x}}^{-1}(\tilde{x}(t)) \\
C(t)=L_{x x}(\tilde{x}(t))-L_{x \dot{x}}(\tilde{x}(t)) L_{\dot{x} \dot{x}}^{-1}(\tilde{x}(t)) L_{\dot{x} x}(\tilde{x}(t)) .
\end{gathered}
$$

3.2 Note: Observe that, if $L \in C^{2}(\mathcal{A})$ and $x \in X(\mathcal{A}) \cap \mathcal{L}^{\prime} \cap C^{1}$, from the theory of linear differential equations applied to $(\mathrm{J})$, any $y \in \mathcal{E}(x)$ is uniquely determined by the values of $y(a), \dot{y}(a)$ or, equivalently, by the values $y(a), q(a)$ at a single point $t=a$ on $T$. In particular, if $y(a)=\dot{y}(a)=0$ then $y \equiv 0$ on $T$. The same conclusion follows, as we shall see below, under weaker assumptions on $L$ and $x$.

Proof of Theorem 2.8: Suppose $x \in \mathcal{H}$ and $s$ in $\left(t_{0}, t_{1}\right)$ is conjugate to $t_{0}$ on $x$. Let $y \in X$ be as in 2.6, that is, $y \in \mathcal{J}(x), y\left(t_{0}\right)=y(s)=0$ and $y \not \equiv 0$ on $\left(t_{0}, s\right)$. Define $z(t):=y(t)$ if $t \in\left[t_{0}, s\right], z(t):=0$ if $t \in\left[s, t_{1}\right]$. Then $z$ belongs to $Y$ and

$I^{\prime \prime}(x ; z)=\int_{t_{0}}^{t_{1}}\left\{\left\langle z(t), \Omega_{y}(\tilde{z}(t))\right\rangle+\left\langle\dot{z}(t), \Omega_{\dot{y}}(\tilde{z}(t))\right\rangle\right\} d t=\int_{t_{0}}^{s} \frac{d}{d t}\left\langle y(t), \Omega_{\dot{y}}(\tilde{y}(t))\right\rangle d t=0$.

Since $x \in \mathcal{H}$, $z$ solves $(\mathrm{AP})$. Since $L \in C^{2}(\mathcal{A})$ and $x \in C^{1}$, the functions $\Omega, \Omega_{y}$, and $\Omega_{\dot{y}}$ are continuous and so, by Theorem 2.1, $z \in \mathcal{E}(x)$. But $z(t)=\dot{z}(t)=0$ for any $t \in\left(s, t_{1}\right)$ and so, by Note $3.2, z \equiv 0$ on $T$. This contradicts the nonvanishing of $y$ and the result follows.

Theorem 2.8 plays a fundamental role in the theory of conjugate points. However, its conclusion, which states that if $I^{\prime \prime}(x ; y) \geq 0$ for all $y \in Y$ then $\left(t_{0}, t_{1}\right)$ contains no conjugate points to $t_{0}$ on $x$, is proved by different authors under different assumptions on both the Lagrangian and the trajectory and, even more, under different definitions of conjugate points. In the proof given above, based on [14], it is assumed that $L \in C^{2}(\mathcal{A})$ and $x \in X(\mathcal{A}) \cap \mathcal{E} \cap \mathcal{L}^{\prime} \cap C^{1}$. Also, a function $y$ corresponding to a point $s \in\left(t_{0}, t_{1}\right]$ conjugate to $t_{0}$ on $x$ is defined on the entire interval $\left[t_{0}, t_{1}\right]$ but should be nonzero on $\left(t_{0}, s\right)$. There are references where $n=1$ ([11], [10], [15], [26]), $L \in C^{4}(\mathcal{A})([6],[11]), L \in C^{3}(\mathcal{A})$ $([12]), L \in C^{2}(\mathcal{A} ; x, \dot{x})([10]), x \in C^{4}([11]), y$ is defined only on $\left[t_{0}, s\right]$ and 
satisfies the so called "ultradifferentiated form" of Jacobi's equation ([10], [15]), and so on. Though the proofs may vary, and some of the assumptions may be crucial throughout the specific proofs, all these references share one feature in common. The trajectory $x$ should belong to $\mathcal{L}^{\prime} \cap C^{1}$.

The importance of $x$ satisfying the strengthened condition of Legendre (and thus being nonsingular) may be inferred from [10, p 64] where it says: "The theory of the Jacobi condition depends upon the hypothesis that the function being examined is nonsingular. If it does, then the Jacobi condition is necessary." The requirement of this assumption is also illustrated, for example, in Proposition 3.1, where $L_{\dot{x} \dot{x}}^{-1}(\tilde{x}(t))$ is assumed to exist for all $t \in T$.

On the other hand, the importance of $x$ being of class $C^{1}$ can be seen in the proof of Theorem 2.8 given above where we have applied the first order necessary condition of Theorem 2.1 to the integrand $\Omega$, and this theorem requires $\Omega, \Omega_{y}$ and $\Omega_{\dot{y}}$ to be continuous. Moreover, the proof invokes Note 3.2 which is valid under the assumption that $x \in C^{1}$. It can also be deduced, for example, from the first lines of [15, p 55]: "Thus far we have considered three necessary conditions for a function $x$ to render a minimum of an integral $I$ on $X$. These conditions are the Euler, Weierstrass, and Legendre conditions [...] Now we turn to a necessary condition for a minimum that must be met by smooth extremals, that is, by extremals without corners; this is Jacobi's necessary condition."

As we shall see below, the assumption $x \in \mathcal{L}^{\prime} \cap C^{1}$ can be weakened and, though the differences may seem to be rather subtle, we shall later discuss some crucial implications in control problems.

It is not the objective of this paper to find out which are the weakest assumptions required for first and second order necessary conditions to hold. However, for our purposes, we shall consider at present the following assumptions on $L$. In what follows, $\mathcal{A}=T \times O \times V$ where $O, V \subset \mathbf{R}^{n}$ are open.

\section{Assumption A1.}

a. $L(t, \cdot, \cdot)$ is $C^{1}(O \times V)$ for all $t \in T$.

b. For all $(x, \dot{x}) \in O \times V, L$ and its first order derivatives in $(x, \dot{x})$ are piecewise continuous in $t$.

c. There exists an integrable function $\alpha: T \rightarrow \mathbf{R}$ such that, for all $(t, x, \dot{x})$ in $T \times O \times V$,

$$
|L(t, x, \dot{x})|+\left|L_{x}(t, x, \dot{x})\right|+\left|L_{\dot{x}}(t, x, \dot{x})\right| \leq \alpha(t)
$$

\section{Assumption A2.}

a. $L(t, \cdot, \cdot)$ is $C^{2}(O \times V)$ for all $t \in T$.

b. For all $(x, \dot{x}) \in O \times V, L$ and its second order derivatives in $(x, \dot{x})$ are piecewise continuous in $t$. 
c. There exists an integrable function $\beta: T \rightarrow \mathbf{R}$ such that, for all $(t, x, \dot{x})$ in $T \times O \times V$,

$$
\left|L_{x x}(t, x, \dot{x})\right|+\left|L_{x \dot{x}}(t, x, \dot{x})\right|+\left|L_{\dot{x} \dot{x}}(t, x, \dot{x})\right| \leq \beta(t) .
$$

From [30, Theorem 5.1] we have the following result (compare with Theorem 2.1 and Note 2.5).

3.3 Theorem: Suppose $x$ solves $\mathrm{P}(\mathcal{A})$. If $(\mathrm{A} 1)$ holds then $M(x) \neq \emptyset$. If also (A2) holds then $x \in \mathcal{H}$.

\section{A change on the assumptions}

Assumptions (A1) and (A2) are simply "recalled" in [30]. Apparently (from the list of references in [30]) the assumptions are taken from [13] where first and second order conditions are obtained for a certain Mayer optimal control problem. On the other hand, some results of [19] play a fundamental role in the techniques employed in [13]. However, in [19], first order necessary conditions are derived (in one of several cases) replacing assumption (A1c) by

$\mathbf{c}^{\prime}$. For each compact subset $O_{c}$ of $O$ and each compact subset $V_{c}$ of $V$, there exists an integrable function $\alpha: T \rightarrow \mathbf{R}$ such that, for all $(t, x, \dot{x})$ in $T \times O_{c} \times V_{c}$

$$
|L(t, x, \dot{x})|+\left|L_{x}(t, x, \dot{x})\right|+\left|L_{\dot{x}}(t, x, \dot{x})\right| \leq \alpha(t)
$$

It is worth mentioning that (see [19, p 283]), for the problem we are dealing with, if $L$ is continuous and continuously differentiable with respect to $(x, \dot{x})$, then $\alpha$ may even be taken to be constant in $\left(\mathrm{c}^{\prime}\right)$. This is certainly not the case with respect to (c). More importantly, when dealing with the accessory problem, the assumption (A1c), with respect to the integrand $\Omega$, may not hold in general, implying that the first order conditions of [13] or [30] may not be applied.

In the remaining of this section we assume that both (A1) and (A2) hold, but replacing (A1c) (and in the obvious way also (A2c)) as above. With this change in the assumptions, as one can verify from the proofs, Theorem 3.3 (based on [13], [19] and [30]) still holds.

Let us now introduce the following notation. For any $x \in X$ and $s \in$ $\left(t_{0}, t_{1}\right]$ let $X_{s}$ be the space of piecewise $C^{1}$ functions mapping $\left[t_{0}, s\right]$ to $\mathbf{R}^{n}$, denote by $Y_{s}$ the set of $y \in X_{s}$ such that $y\left(t_{0}\right)=y(s)=0$, and let $\mathcal{J}_{s}(x)$ be the set of $y \in X_{s}$ satisfying

$$
\frac{d}{d t}\left[L_{\dot{x} x}(\tilde{x}(t)) y(t)+L_{\dot{x} \dot{x}}(\tilde{x}(t)) \dot{y}(t)\right]=L_{x x}(\tilde{x}(t)) y(t)+L_{x \dot{x}}(\tilde{x}(t)) \dot{y}(t) \quad\left(t \in\left[t_{0}, s\right]\right) .
$$


3.4 Definition: For any $x \in X$ let $\mathcal{P}(x)$ be the set of points $s \in\left(t_{0}, t_{1}\right]$ for which there exist $y \in X_{s}$ and $\epsilon>0$ such that

i. $y \in Y_{s} \cap \mathcal{J}_{s}(x)$ with $y \not \equiv 0$ on $[s-\epsilon, s] \cap T$.

ii. $L_{\dot{x} \dot{x}}(\tilde{x}(t))>0$ for all $t \in[s-\epsilon, s+\epsilon] \cap T$.

Note that, if $x \in \mathcal{L}^{\prime}$, we have $\mathcal{C}(x) \subset \mathcal{P}(x)$ (simply take $\epsilon=s-t_{0}$ ). The improved version of Theorem 2.8 we wish to establish is the following.

3.5 Theorem: Let $x \in X(\mathcal{A})$. Then $x \in \mathcal{H} \Rightarrow \mathcal{P}(x) \cap\left(t_{0}, t_{1}\right)=\emptyset$.

Proof: Suppose $x \in \mathcal{H}$ and there exists $s \in \mathcal{P}(x) \cap\left(t_{0}, t_{1}\right)$. Let $y \in X_{s}$ and $\epsilon>0$ be as in 3.4, and define $z(t):=y(t)$ if $t \in\left[t_{0}, s\right], z(t):=0$ if $t \in\left[s, t_{1}\right]$. Then $z$ solves (AP) and, by Theorem 3.3, $\tilde{M}(z) \neq \emptyset$. Let $r \in \tilde{M}(z)$. Since $s \in$ $\left(t_{0}, t_{1}\right)$ we can suppose, without loss of generality, that $\epsilon<\min \left\{s-t_{0}, t_{1}-s\right\}$. Observe that $(z, r)$ satisfies $(\mathrm{J})$ on $[s-\epsilon, s+\epsilon]$, and Note 3.2 (applied on this interval) is still valid under our assumptions on $L$ and $x$ (the functions $A, B$, $C$ of 3.1 are now piecewise continuous on $[s-\epsilon, s+\epsilon])$. Since $z(t)=\dot{z}(t)=0$ for any $t \in(s, s+\epsilon)$, we have $z \equiv 0$ on $[s-\epsilon, s+\epsilon]$, contradicting that $y \neq \equiv$ on $[s-\epsilon, s]$.

\section{The continuity approach}

Let us turn now to a different approach which can be found, for example, in [1], [9], [18]. According to [1], a point $s$ is conjugate to $t_{0}$ (with respect to the functional $\left.I^{\prime \prime}(x ; \cdot)\right)$ if there exists $y \in \mathcal{J}(x)$ with $y\left(t_{0}\right)=y(s)=0$ and $L_{\dot{x} \dot{x}}(\tilde{x}(s)) \dot{y}(s) \neq 0$. Theorem 2.7 is established in [1] under the assumptions that $L \in C^{2}(\mathcal{A} ; x, \dot{x})$ and $X$, the space of trajectories, is $C^{1}\left(T, \mathbf{R}^{n}\right)$. In [9], conjugacy is defined for the case $n=1$ and trajectories $x$ in $\mathcal{E} \cap C^{4}$. A point $s$ is called conjugate of $t_{0}$ for the extremal $x$ if there is a function $y \in \mathcal{J}_{s}(x)$ of class $C^{2}$ which satisfies $y\left(t_{0}\right)=y(s)=0$, and which on each subinterval of positive length of its domain of definition is not identically zero. It is then proved that, if $L \in C^{3}(\mathcal{A})$ and $x \in C^{4}$ solves $\mathrm{P}(\mathcal{A})$, a point $s \in\left(t_{0}, t_{1}\right)$ can only be a conjugate point of $t_{0}$ if $L_{\dot{x} \dot{x}}(\tilde{x}(s))=0$. A slightly different but similar definition is given in [18] where it is proved that, if $L, L_{x}$ and $L_{\dot{x}}$ are of class $C^{2}(\mathcal{A})$ and $x \in C^{2}$ solves $\mathrm{P}(\mathcal{A})$, there can be no point $s \in\left(t_{0}, t_{1}\right)$ conjugate to $t_{0}$ on $x$ satisfying $\left|L_{\dot{x} \dot{x}}(\tilde{x}(s))\right| \neq 0$.

Based on this second approach, let us define the following set of points.

3.6 Definition: For any $x \in X$ let $\mathcal{Q}(x)$ be the set of points $s \in\left(t_{0}, t_{1}\right]$ for which there exists $y \in Y_{s} \cap \mathcal{J}_{s}(x)$ with $L_{\dot{x} \dot{x}}(\tilde{x}(s)) \dot{y}(s) \neq 0$.

Note that $x \in \mathcal{L}^{\prime} \Rightarrow \mathcal{Q}(x) \subset \mathcal{P}(x)$. The converse also holds, under the classical assumptions $L \in C^{2}(\mathcal{A})$ and $x \in X(\mathcal{A}) \cap \mathcal{L}^{\prime} \cap C^{1}$, if we deal with $\mathcal{E}(x)$ instead of $\mathcal{J}(x)$. There are texts where this modification occurs explicitly (see, for example, [16]) or implicitly (in [6], [9], [11], [12], for example, the 
assumptions imply that $\mathcal{E}(x)=\mathcal{J}(x)$ ). The theory of [1], [9] and [18], showing that Jacobi's condition is necessary for optimality, is a consequence of the following result.

3.7 Theorem: Let $x \in X(\mathcal{A})$. Then $x \in \mathcal{H} \Rightarrow \mathcal{Q}(x) \cap\left(t_{0}, t_{1}\right)=\emptyset$.

Proof: Suppose $x \in \mathcal{H}$ and there exists $s \in \mathcal{Q}(x) \cap\left(t_{0}, t_{1}\right)$. Let $y \in X_{s}$ be as in 3.6, and define $z(t):=y(t)$ if $t \in\left[t_{0}, s\right], z(t):=0$ if $t \in\left[s, t_{1}\right]$. Then $z$ solves $(\mathrm{AP})$. By Theorem 3.3, $\tilde{M}(z) \neq \emptyset$. Let $r \in \tilde{M}(z)$ and note that $r(s)=L_{\dot{x} \dot{x}}(\tilde{x}(s)) \dot{z}(s)$ and $r$ is continuous. But $r(s-) \neq 0=r(s+)$, and we reach a contradiction.

\section{Points conjugate to $t_{1}$}

Up to this point we have dealt with points conjugate to $t_{0}$, the initial point of the time interval. We have done so because this is the way Jacobi's necessary condition is presented in all the references treating the calculus of variations problem that we have mentioned ([1], [6], [9], [10], [11], [12], [14], [15], [16], [18], [26]). In [30], however, conjugate points (for certain classes of optimal control problems) are defined with respect to the final point of the interval. Since we shall be interested in comparing that definition with those given in this section, let us also consider this case. Though this modification is trivial in calculus of variations, as we show next, several inequalities appearing in more complicated problems make it rather cumbersome.

Let us first redefine the sets $X_{s}$ and $\mathcal{J}_{s}$ exactly as before but with $\left[s, t_{1}\right]$ instead of $\left[t_{0}, s\right]$, and for points $s \in\left[t_{0}, t_{1}\right)$ instead of $\left(t_{0}, t_{1}\right]$. The space $Y_{s}$ will now denote the set of $y \in X_{s}$ satisfying $y(s)=y\left(t_{1}\right)=0$. Define $\mathcal{P}(x)$ and $\mathcal{Q}(x)$ as in 3.4 and 3.6 (for points $s \in\left[t_{0}, t_{1}\right)$ ), replacing condition (i) of 3.4 by

i. $y \in Y_{s} \cap \mathcal{J}_{s}(x)$ with $y \not \equiv 0$ on $[s, s+\epsilon] \cap T$.

One readily verifies that, with proofs similar to those given above, Theorems 3.5 and 3.7 remain valid.

We end this section with a simple example, not covered by the classical theory, for which the new sets introduced above can be applied.

3.8 Example: Let $a>0$ and consider the problem of minimizing

$$
I(x)=\frac{1}{2} \int_{0}^{2 a} \varphi(t)\left\{\dot{x}^{2}(t)-x^{2}(t)\right\} d t
$$

subject to $x(0)=x(2 a)=0$, where $\varphi(t)=t$ if $t \in[0, a], \varphi(t)=2 a-t$ if $t \in[a, 2 a]$.

In this case $n=1, T=[0,2 a], \xi_{0}=\xi_{1}=0, \mathcal{A}=T \times \mathbf{R}^{2}$, and $L(t, x, \dot{x})=$ $\varphi(t)\left(\dot{x}^{2}-x^{2}\right) / 2$.

Note first that $L_{\dot{x} \dot{x}}(\tilde{x}(t))=\varphi(t)$ for all $t \in T$ and so any trajectory is singular. Thus the first approach, in the classical context, cannot be applied. 
The same occurs with the second one since $L$ is not even of class $C^{1}$. Observe also that Jacobi's equation, with respect to any trajectory $x$, is given by

$$
\frac{d}{d t}[\varphi(t) \dot{y}(t)]=-\varphi(t) y(t) \quad(t \in T) .
$$

For points in $[0, a]$, this corresponds (for $y$ of class $C^{2}$ ) to Bessel's equation of order zero, which is given by $t \ddot{y}(t)+\dot{y}(t)+t y(t)=0$. If $J_{0}$ and $Y_{0}$ denote, respectively, the Bessel functions of the first and second kind of order zero, the general solution (see, for example, [7]) is $y(t)=c_{1} J_{0}(t)+c_{2} Y_{0}(t)$, which is valid for all finite $t$ excepting $Y_{0}(t)$ as $t \rightarrow 0$. This function then ceases to be a solution, and we are left with $c_{1} J_{0}(t)$. Since $J_{0}(0)=1$, the only $\left(C^{2}\right)$ solution vanishing at $t=0$ is $y_{0} \equiv 0$. In particular, this fact rules out all points in $(0, a]$, in terms of any of the two classical approaches, as points conjugate to $t=0$.

Let us now apply our results. Let $x_{0} \equiv 0$. Note that, since the second variation with respect to any trajectory $x$ is given by $I^{\prime \prime}(x ; y)=\int_{0}^{a} \varphi(t)\left\{\dot{y}^{2}(t)-\right.$ $\left.y^{2}(t)\right\} d t$, we have $\mathcal{H} \neq \emptyset$ if and only if $x_{0}$ solves $\mathrm{P}(\mathcal{A})$.

Let $\eta \simeq 2.4048$ be the first zero of $J_{0}(t)(t>0)$ and let $\zeta_{0} \simeq 0.8935$ and $\zeta_{1} \simeq 3.9576$ be the first two zeros of $Y_{0}(t)(t>0)$. We shall consider only the case $a>\eta$. Fix a point $\eta<\tau<\min \left\{a, \zeta_{1}\right\}$ and define

$$
F(t):=J_{0}(\tau) Y_{0}(t)-J_{0}(t) Y_{0}(\tau) \quad \text { for all } t \in\left(0, \zeta_{0}\right] .
$$

Note that $J_{0}\left(\zeta_{0}\right)>0$ and, since $\tau \in\left(\eta, \zeta_{1}\right), J_{0}(\tau)<0<Y_{0}(\tau)$. Thus

$$
F\left(\zeta_{0}\right)=-J_{0}\left(\zeta_{0}\right) Y_{0}(\tau)<0 \quad \text { and } \quad \lim _{t \rightarrow 0} F(t)=+\infty
$$

Since $F$ is continuous, there exists $s \in\left(0, \zeta_{0}\right)$ such that $F(s)=0$. Let

$$
c:=\frac{J_{0}(s)}{Y_{0}(s)}=\frac{J_{0}(\tau)}{Y_{0}(\tau)} \quad \text { and } \quad y(t):= \begin{cases}J_{0}(t)-c Y_{0}(t) & \text { if } t \in[s, \tau] \\ 0 & \text { if } t \in[\tau, 2 a]\end{cases}
$$

Hence $y(s)=y(2 a)=0$ and $y$ satisfies Jacobi's equation on $[s, 2 a]$, that is, $y \in Y_{s} \cap \mathcal{J}_{s}(x)$ (note that $y \notin \mathcal{E}(x)$ ). Since $s>0$, we can choose $\epsilon>0$ such that $L_{\dot{x} \dot{x}}(\tilde{x}(t))=t>0$ for all $t \in[s-\epsilon, s+\epsilon]$ and $y \not \equiv 0$ on $[s, s+\epsilon]$, showing that $s \in \mathcal{P}(x) \cap(0,2 a)$. By Theorem 3.5, the problem (with $a>\eta$ ) has no solution (this also follows by Theorem 3.7 since $s \dot{y}(s) \neq 0$ and so $s \in \mathcal{Q}(x) \cap(0,2 a)$ ).

\section{Fixed-endpoint control problem: necessity}

According to Gilbert and Bernstein [13], the earliest mathematically rigorous treatment of second order necessary conditions in optimal control, motivated by the work of Bliss [6] in the calculus of variations, is due to Hestenes 
[14]. Since then, this question has been studied by a large number of authors. In the literature, one can now find second order conditions for a wide variety of different specific optimal control problems according to the constraints, the spaces of admissible processes, the assumptions on the functions delimiting the problem, and so on. Not all coincide, and it may be extremely cumbersome to compare between different problems and the conditions obtained, but this is not the issue of this paper.

As mentioned in the introduction, we are mainly interested in finding an "appropriate" notion of conjugate points for certain optimal control problems. As in Section 2, for the basic problem in calculus of variations, our purpose in this section will be to provide the reader with what we can call a "classical approach" to second order conditions. Both the statement and the proof will serve us as a point of reference. For simplicity of exposition and to keep notational complexity to a minimum, we shall deal with the fixed-endpoint control problem of Lagrange (without isoperimetric constraints), as presented in [14, p 251]. In this section, based on the approach given in [14, Chapter 6], we state first and second order necessary conditions which generalize those of Section 2. The notation will be similar to the one used before, but no confusion should arise.

Suppose we are given an interval $T:=\left[t_{0}, t_{1}\right]$ in $\mathbf{R}$, two points $\xi_{0}, \xi_{1}$ in $\mathbf{R}^{n}$, a set $\mathcal{A}$ in $T \times \mathbf{R}^{n} \times \mathbf{R}^{m}$, and functions $L$ and $f$ mapping $T \times \mathbf{R}^{n} \times \mathbf{R}^{m}$ to $\mathbf{R}$ and $\mathbf{R}^{n}$ respectively. Denote by $X$ the space of piecewise $C^{1}$ functions mapping $T$ to $\mathbf{R}^{n}$, by $\mathcal{U}$ the space of piecewise continuous functions mapping $T$ to $\mathbf{R}^{m}$, set $Z:=X \times \mathcal{U}$,

$$
\begin{gathered}
D:=\{(x, u) \in Z \mid \dot{x}(t)=f(t, x(t), u(t)) \quad(t \in T)\}, \\
Z(\mathcal{A}):=\{(x, u) \in Z \mid(t, x(t), u(t)) \in \mathcal{A} \quad(t \in T)\}, \\
Z_{e}(\mathcal{A}):=\left\{(x, u) \in D \cap Z(\mathcal{A}) \mid x\left(t_{0}\right)=\xi_{0}, x\left(t_{1}\right)=\xi_{1}\right\},
\end{gathered}
$$

and consider the functional $I: Z \rightarrow \mathbf{R}$ given by $I(x, u):=\int_{t_{0}}^{t_{1}} L(t, x(t), u(t)) d t$ $((x, u) \in Z)$. The problem we shall deal with, which we label $\mathrm{P}(\mathcal{A})$, is that of minimizing $I$ over $Z_{e}(\mathcal{A})$.

A common and concise way of formulating this problem is as follows:

Minimize $I(x, u)=\int_{t_{0}}^{t_{1}} L(t, x(t), u(t)) d t$ subject to
a. $(x, u) \in X \times \mathcal{U}$;
b. $\dot{x}(t)=f(t, x(t), u(t))(t \in T)$;
c. $x\left(t_{0}\right)=\xi_{0}, x\left(t_{1}\right)=\xi_{1}$;
d. $(t, x(t), u(t)) \in \mathcal{A}(t \in T)$.

Elements of $Z$ will be called processes, and a process $(x, u)$ solves $\mathrm{P}(\mathcal{A})$ if $(x, u) \in Z_{e}(\mathcal{A})$ and $I(x, u) \leq I(y, v)$ for all $(y, v) \in Z_{e}(\mathcal{A})$. For local minima we could proceed as in Section 2 but, dealing only with necessity, no difficulties 
arise in extending the theory to follow to weak or strong local optimality. We shall therefore consider only the global case.

For any $(x, u) \in Z$ we shall use the notation $(\tilde{x}(t))$ to represent $(t, x(t), u(t))$. We assume, unless otherwise specified, that the functions $L, f$ are of class $C^{2}(\mathcal{A})$, and $\mathcal{A}$ is open.

Let us summarize the necessary conditions we are about to obtain. We begin with a few definitions.

- For all $(t, x, u, p, \lambda)$ in $T \times \mathbf{R}^{n} \times \mathbf{R}^{m} \times \mathbf{R}^{n} \times \mathbf{R}$ let

$$
H(t, x, u, p, \lambda):=\langle p, f(t, x, u)\rangle-\lambda L(t, x, u) .
$$

- For all $(x, u) \in Z$ let $A(t):=f_{x}(\tilde{x}(t)), B(t):=f_{u}(\tilde{x}(t))(t \in T)$, and define

$$
D(x, u):=\{(y, v) \in Z \mid \dot{y}(t)=A(t) y(t)+B(t) v(t)(t \in T)\} .
$$

The set of admissible variations along $(x, u)$ will be given by

$$
Y(x, u):=\left\{(y, v) \in D(x, u) \mid y\left(t_{0}\right)=y\left(t_{1}\right)=0\right\} .
$$

- A process $(x, u)$ will be said to be normal to $\mathrm{P}(\mathcal{A})$ if there exist $\left(y_{1}, v_{1}\right), \ldots,\left(y_{n}, v_{n}\right) \in D(x, u)$ with $y_{i}\left(t_{0}\right)=0(i=1, \ldots, n)$ such that $\left|y_{1}\left(t_{1}\right) \cdots y_{n}\left(t_{1}\right)\right| \neq 0$.

- For all $(x, u) \in Z$ let

$M(x, u):=\left\{\left(\lambda_{0}, p\right) \in \mathbf{R} \times X\left|\lambda_{0} \geq 0, \lambda_{0}+\right| p \mid \neq 0, \dot{p}(t)=-H_{x}^{*}(t), H_{u}(t)=0(t \in T)\right\}$

where $H(t)$ denotes $H\left(\tilde{x}(t), p(t), \lambda_{0}\right)$, and consider the following sets:

$$
\begin{gathered}
\mathcal{E}:=\{(x, u, p) \in Z \times X \mid(x, u) \in D \text { and }(1, p) \in M(x, u)\} \\
\mathcal{H}:=\left\{(x, u, p) \in Z \times X \mid J_{p}((x, u) ;(y, v)) \geq 0 \text { for all }(y, v) \in Y(x, u)\right\}
\end{gathered}
$$

where

$$
J_{p}((x, u) ;(y, v))=\int_{t_{0}}^{t_{1}} 2 \Omega(t, y(t), v(t)) d t \quad((y, v) \in Z)
$$

and, for all $(t, y, v) \in T \times \mathbf{R}^{n} \times \mathbf{R}^{m}$,

$$
\begin{gathered}
2 \Omega(t, y, v):= \\
-\left[\left\langle y, H_{x x}(\tilde{x}(t), p(t), 1) y\right\rangle+2\left\langle y, H_{x u}(\tilde{x}(t), p(t), 1) v\right\rangle+\left\langle v, H_{u u}(\tilde{x}(t), p(t), 1) v\right\rangle\right] .
\end{gathered}
$$

The following theorem provides first and second order conditions for problem $\mathrm{P}(\mathcal{A})$. These conditions can be written in a succinct way in terms of $\mathcal{E}$ and $\mathcal{H}$. 
4.1 Theorem: Suppose $(x, u)$ solves $\mathrm{P}(\mathcal{A})$. Then $M(x, u) \neq \emptyset$. If $(x, u)$ is normal to $\mathrm{P}(\mathcal{A})$ then there exists a unique $p \in X$ such that $(x, u, p) \in \mathcal{E}$. Moreover, $(x, u, p) \in \mathcal{H}$.

It is a common practice to state results of this nature by giving certain names to the assumptions, conditions, and functions involved. In contrast, we have chosen this notation not only because it allows us to express the results in a concise way but, mainly, to avoid any misleading interpretations. The same words or phrases can have completely different meanings in different texts, sometimes even by the same author.

To give an example, $\left(x, u, p, \lambda_{0}\right)$ is called an extremal in [14] if $(x, u) \in D$, $\left(\lambda_{0}, p\right) \in M(x, u)$, and $u$ is continuous. In [27], an extremal is a couple satisfying Pontryagin's minimum principle. In [28], an extremal is a pair $(x, u) \in Z_{e}(\mathcal{A})$ for which $M(x, u) \neq \emptyset$. Such a pair is called a regular extremal in [30]. In [17], a pair $(x, u) \in Z_{e}(\mathcal{A})$ is an extremal if there exists an arc $p$ (meaning an absolutely continuous function mapping $T$ to $\mathbf{R}^{n}$ ) such that $(1, p) \in M(x, u)$. Such a pair, with $p \in X$, is called weakly normal in [29].

Now, we are interested in showing how the second order condition " $(x, u, p) \in$ $\mathcal{H}$ " can be easily established. To do so, let us state three auxiliary results from [14]. The first one (maximum principle) is proved in [14, p 254] under the following assumptions on the data of the problem.

B: The functions $L, f$ and their partial derivatives with respect to $x$ are continuous on $\mathcal{A}$. The set $\mathcal{A}$ is admissible in the sense that, for each $(s, y, v) \in \mathcal{A}$, there exist $\delta>0$ and $u:[s-\delta, s+\delta] \rightarrow \mathbf{R}^{m}$ continuous such that $u(s)=v$ and $(t, x, u(t)) \in \mathcal{A}$ for all $(t, x) \in T \times \mathbf{R}^{n}$ with $|(t, x)-(s, y)|<\delta$.

4.2 Theorem: Assume (B) holds. If $\left(x_{0}, u_{0}\right)$ solves $\mathrm{P}(\mathcal{A})$ then there exist $\lambda_{0} \geq 0$ and $p \in X$, not both zero, such that

a. $\dot{p}(t)=-H_{x}^{*}\left(\tilde{x}_{0}(t), p(t), \lambda_{0}\right)$ on every interval of continuity of $u_{0}$.

b. $H\left(t, x_{0}(t), u, p(t), \lambda_{0}\right) \leq H\left(\tilde{x}_{0}(t), p(t), \lambda_{0}\right)$ for all $(t, u) \in T \times \mathbf{R}^{m}$ with $\left(t, x_{0}(t), u\right) \in \mathcal{A}$.

4.3 Note: In Theorem 4.2, the function $t \mapsto H\left(t, x_{0}(t), u_{0}(t), p(t), \lambda_{0}\right)$ is continuous on $T$ and, if $\mathcal{A}$ is open, then $L, f \in C^{1}(\mathcal{A} ; u) \Rightarrow H_{u}\left(\tilde{x}_{0}(t), p(t), \lambda_{0}\right)=$ 0 , and $L, f \in C^{2}(\mathcal{A} ; u) \Rightarrow H_{u u}\left(\tilde{x}_{0}(t), p(t), \lambda_{0}\right) \leq 0(t \in T)$.

4.4 Lemma: Suppose $\left(x_{0}, u_{0}\right) \in Z_{e}(\mathcal{A})$ is normal to $\mathrm{P}(\mathcal{A})$ and $(y, v) \in$ $Y\left(x_{0}, u_{0}\right)$. Then there exist $\delta>0$ and a one-parameter family $(x(\cdot, \epsilon), u(\cdot, \epsilon)) \in$ $Z_{e}(\mathcal{A})(|\epsilon|<\delta)$ such that

i. $x(t, 0)=x_{0}(t), u(t, 0)=u_{0}(t)(t \in T)$.

ii. $x_{\epsilon}(t, 0)=y(t), u_{\epsilon}(t, 0)=v(t)(t \in T)$. 
4.5 Note: A process $(x, u)$ is normal to $\mathrm{P}(\mathcal{A})$ if and only if the equations

$$
\begin{gathered}
\dot{p}(t)=-A^{*}(t) p(t) \quad\left[=-H_{x}^{*}(\tilde{x}(t), p(t), 0)\right] \\
0=B^{*}(t) p(t) \quad\left[=H_{u}^{*}(\tilde{x}(t), p(t), 0)\right]
\end{gathered}
$$

have no nonnull solution $p$ on $T$. If $\left(x_{0}, u_{0}\right)$ solves $\mathrm{P}(\mathcal{A})$ and $\left(x_{0}, u_{0}\right)$ is normal to $\mathrm{P}(\mathcal{A})$ then, in Theorem 4.2, $\lambda_{0}>0$. The multipliers $\lambda_{0}, p$ can be chosen so that $\lambda_{0}=1$ and, when so chosen, they are unique.

Proof of Theorem 4.1: Suppose $\left(x_{0}, u_{0}\right)$ solves $\mathrm{P}(\mathcal{A})$. The fact that $M\left(x_{0}, u_{0}\right) \neq \emptyset$ follows by 4.2 and 4.3. If $\left(x_{0}, u_{0}\right)$ is normal to $\mathrm{P}(\mathcal{A})$ then, by 4.5 , there exists a unique $p \in X$ such that $\left(x_{0}, u_{0}, p\right) \in \mathcal{E}$. It remains to show that $\left(x_{0}, u_{0}, p\right) \in \mathcal{H}$. To do so, define

$$
K(x, u):=\left\langle p\left(t_{1}\right), \xi_{1}\right\rangle-\left\langle p\left(t_{0}\right), \xi_{0}\right\rangle+\int_{t_{0}}^{t_{1}} F(t, x(t), u(t)) d t \quad((x, u) \in Z)
$$

where, for all $(t, x, u) \in T \times \mathbf{R}^{n} \times \mathbf{R}^{m}$,

$$
F(t, x, u):=L(t, x, u)-\langle p(t), f(t, x, u)\rangle-\langle\dot{p}(t), x\rangle .
$$

Observe that

$$
F(t, x, u)=-H(t, x, u, p(t), 1)-\langle\dot{p}(t), x\rangle
$$

and, if $(x, u) \in Z_{e}(\mathcal{A}), K(x, u)=I(x, u)$. Let $(y, v) \in Y\left(x_{0}, u_{0}\right)$ and let $(x(\cdot, \epsilon), u(\cdot, \epsilon)) \in Z_{e}(\mathcal{A})(|\epsilon|<\delta)$ be a one-parameter family satisfying 4.4 . Hence

$$
g(\epsilon):=K(x(\cdot, \epsilon), u(\cdot, \epsilon))=I(x(\cdot, \epsilon), u(\cdot, \epsilon)) \quad(|\epsilon|<\delta)
$$

satisfies $g(\epsilon) \geq g(0)=K\left(x_{0}, u_{0}\right)=I\left(x_{0}, u_{0}\right)(|\epsilon|<\delta)$. Note that

$$
\begin{gathered}
F_{x}\left(\tilde{x}_{0}(t)\right)=L_{x}\left(\tilde{x}_{0}(t)\right)-p^{*}(t) f_{x}\left(\tilde{x}_{0}(t)\right)-\dot{p}^{*}(t)=0, \\
F_{u}\left(\tilde{x}_{0}(t)\right)=-H_{u}\left(\tilde{x}_{0}(t), p(t), 1\right)=0
\end{gathered}
$$

and so $0=g^{\prime}(0)=K^{\prime}\left(\left(x_{0}, u_{0}\right) ;(y, v)\right)$ and $0 \leq g^{\prime \prime}(0)=K^{\prime \prime}\left(\left(x_{0}, u_{0}\right) ;(y, v)\right)=$ $J_{p}\left(\left(x_{0}, u_{0}\right) ;(y, v)\right)$.

\section{Weaker assumptions and "classical" approaches to conjugacy}

For the fixed-endpoint control problem stated in the previous section, the necessary conditions obtained by Hestenes in [14] are summarized in Theorem 4.1. No attempts are made in this text to characterize the second order condition in terms of a generalized notion of conjugate points.

One of the first attempts in this direction was made by Zeidan and Zezza [30]. There, the optimal control problem considered can be stated as follows. 
Given an interval $T=\left[t_{0}, t_{1}\right]$, a point $\xi_{0} \in \mathbf{R}^{n}$, sets $O \subset \mathbf{R}^{n}$ and $V \subset \mathbf{R}^{m}$ open, and functions $g: O \rightarrow \mathbf{R},(f, L): T \times O \times V \rightarrow \mathbf{R}^{n} \times \mathbf{R}, \varphi: V \rightarrow \mathbf{R}^{r}$ $(r \leq m), h: O \rightarrow \mathbf{R}^{k}(k \leq n)$,

minimize $I(x, u)=g\left(x\left(t_{1}\right)\right)+\int_{t_{0}}^{t_{1}} L(t, x(t), u(t)) d t$ subject to

a. $(x, u) \in X \times \mathcal{U}$;

b. $\dot{x}(t)=f(t, x(t), u(t))(t \in T)$;

c. $x\left(t_{0}\right)=\xi_{0}, x\left(t_{1}\right) \in C$;

d. $(t, x(t), u(t)) \in \mathcal{A}(t \in T)$

where $X$ is the space of piecewise $C^{1}$ functions mapping $T$ to $O, \mathcal{U}$ is the space of piecewise continuous functions mapping $T$ to $V, C=\left\{x \in \mathbf{R}^{n} \mid\right.$ $h(x)=0\}$, and $\mathcal{A}=\{(t, x, u) \in T \times O \times V \mid \varphi(u)=0\}$.

For this problem, necessary conditions like those of Theorem 4.1 are obtained in [30] essentially in the same way as in [14], though under weaker assumptions. The corresponding Theorem 4.2 (as a minimum principle), Lemma 4.4 and Note 4.5 are, as before, the main auxiliary results used in their proof. Then, a notion of conjugate points is introduced and, assuming nonsingularity of the process of interest together with certain normality conditions, it is proved that a necessary condition for optimality is the nonexistence of such points in the open interval.

Since we are primarily interested in the approach to conjugacy, we shall continue, for simplicity of notation, dealing with the fixed-endpoint problem $\mathrm{P}(\mathcal{A})$ of Section 4. The weaker assumptions imposed in [30] correspond to (A1) and (A2) of Section 3 with $L$ replaced by the function $(f, L)$ and $\dot{x}$ by $u$. Theorem 5.1 of [30], when reduced to our problem, yields the following result.

5.1 Theorem: Suppose (A1) holds and $(x, u)$ solves $\mathrm{P}(\mathcal{A})$. If $(x, u)$ is normal to $\mathrm{P}(\mathcal{A})$, there exists a unique $p \in X$ such that $(x, u, p) \in \mathcal{E}$. If, in addition, (A2) holds, then $(x, u, p) \in \mathcal{H}$.

In the remaining of this paper we assume that (A1) and (A2), as modified in Section 3, hold.

With the idea of giving a clear explanation of the notion of conjugacy introduced in [30], let us make a few observations analogous to those of Section 3. Recall that, given $(x, u) \in Z$, we let $A(t):=f_{x}(\tilde{x}(t))$ and $B(t):=f_{u}(\tilde{x}(t))$ $(t \in T)$.

- For any $(x, u, p) \in Z \times X$ consider the accessory problem (with respect to $(x, u, p))$, which we label $(\operatorname{AP})$, of minimizing $J_{p}((x, u) ; \cdot) / 2$ over $Y(x, u)$, that is,

minimize $K(y, v):=\int_{t_{0}}^{t_{1}} \Omega(t, y(t), v(t)) d t$ subject to

a. $(y, v) \in X \times \mathcal{U}$;

b. $\dot{y}(t)=A(t) y(t)+B(t) v(t)(t \in T)$; 
c. $y\left(t_{0}\right)=y\left(t_{1}\right)=0$;

d. $(t, y(t), v(t)) \in T \times \mathbf{R}^{n} \times \mathbf{R}^{m}(t \in T)$.

- Given $(x, u, p) \in Z \times X$, define

$$
\tilde{H}(t, y, v, q, \lambda):=\langle q, A(t) y+B(t) v\rangle-\lambda \Omega(t, y, v)
$$

and let $\tilde{M}(y, v)$ be the set of pairs $\left(\lambda_{0}, q\right)$ in $\mathbf{R} \times X$ satisfying $\lambda_{0} \geq 0, \lambda_{0}+|q| \neq 0$, and

$$
\begin{gathered}
\dot{q}(t)=-\tilde{H}_{y}^{*}\left(\tilde{y}(t), q(t), \lambda_{0}\right) \quad\left[=-A^{*}(t) q(t)+\lambda_{0} \Omega_{y}^{*}(\tilde{y}(t))\right], \\
0=\tilde{H}_{v}^{*}\left(\tilde{y}(t), q(t), \lambda_{0}\right) \quad\left[=B^{*}(t) q(t)-\lambda_{0} \Omega_{v}^{*}(\tilde{y}(t))\right] .
\end{gathered}
$$

- Following the classical approach in the calculus of variations, define a set of "secondary extremals" by

$$
\mathcal{E}(x, u, p):=\{(y, v, q) \in Z \times X \mid(y, v) \in D(x, u) \text { and }(1, q) \in \tilde{M}(y, v)\} .
$$

Note that $(y, v, q) \in Z \times X$ belongs to $\mathcal{E}(x, u, p)$ if and only if, for all $t \in T$,

$$
\begin{gathered}
\dot{y}(t)=A(t) y(t)+B(t) v(t) \\
\dot{q}(t)+A^{*}(t) q(t)=-H_{x x}(\tilde{x}(t), p(t), 1) y(t)-H_{x u}(\tilde{x}(t), p(t), 1) v(t) \\
B^{*}(t) q(t)=-H_{u x}(\tilde{x}(t), p(t), 1) y(t)-H_{u u}(\tilde{x}(t), p(t), 1) v(t) .
\end{gathered}
$$

Based on these definitions let us introduce a set which, in some sense, can be seen as a set of "generalized conjugate points." It should be noted that, when reduced to the calculus of variations problem, there is a slight difference with the set of points given in Definition 2.6 since we are now considering functions $y$ belonging to $\mathcal{E}(x)$ instead of $\mathcal{J}(x)$ (see Section 3 ).

5.2 Definition: For any $(x, u, p) \in Z \times X$ let $\mathcal{C}(x, u, p)$ be the set of points $s \in\left[t_{0}, t_{1}\right)$ for which there exists $(y, v, q) \in \mathcal{E}(x, u, p)$ with $y(s)=y\left(t_{1}\right)=$ 0 and $(y, v, q) \not \equiv(0,0,0)$.

In [30], for $(x, u, p) \in \mathcal{E}$, the elements of $\mathcal{C}(x, u, p)$ are called points conjugate to $t_{1}$ [on or with respect to $\left.(x, u, p)\right]$. A similar definition is given in [31] for a problem slightly different to that of [30]. There, the problem is as before except that $X$ corresponds to the space of absolutely continuous functions mapping $T$ to $O, \mathcal{U}$ is the space of measurable functions mapping $T$ to $V$, and $\mathcal{A}=T \times O \times U$ with $U \subset V$ convex.

One might be tempted to think that a necessary condition for optimality, if $(x, u, p)$ belongs to

$$
\mathcal{L}^{\prime}:=\left\{(x, u, p) \in Z \times X \mid H_{u u}(\tilde{x}(t), p(t), 1)<0 \text { for all } t \in T\right\}
$$

(and thus it is nonsingular (see [14, p 256]) in the sense that $\left|H_{u u}(\tilde{x}(t), p(t), 1)\right| \neq$ $0)$ is, as in the classical theory, the nonexistence of such points in the open 
interval $\left(t_{0}, t_{1}\right)$. It is a trivial fact to show that this is not the case, even assuming normality of the extremal under consideration.

This is illustrated in [32] with three examples. The aim of this note, according to the authors, "is to show how two attempts to extend to the optimal control setting the classical results in the calculus of variations turn out to be incorrect." Bernhard replies in the same journal that "the so-called counterexamples pertaining to the necessary controllability assumptions are at best remarks that these assumptions are not explicit enough in the two references [5] and [8]."

Instead of finding out whose claim is correct, it seems more constructive trying to understand why there are problems for which, with respect to a nonsingular normal solution, the set of conjugate points (as defined above) may be nonempty. The following two examples are taken up from [32].

5.3 Example: Minimize $I(x, u)=\frac{1}{2} \int_{0}^{2} u^{2}(t) d t$ subject to $\dot{x}(t)=\varphi(t) u(t)$ $(t \in[0,2]), x(0)=x(2)=0$, where $\varphi:[0,2] \rightarrow \mathbf{R}$ is a continuous function such that $\varphi(t)>0$ for $t \in[0,1)$ and $\varphi(t)=0$ for $t \in[1,2]$.

In this case, $T=[0,2], \xi_{0}=\xi_{1}=0, \mathcal{A}=T \times \mathbf{R}^{2}, f(t, x, u)=\varphi(t) u$, $L(t, x, u)=u^{2} / 2$.

Note that any $(x, u) \in Z$ is normal to $\mathrm{P}(\mathcal{A})$ since $p \equiv 0$ is the only solution to $\dot{p}(t)=0, \varphi(t) p(t)=0(t \in T)$, and any $(x, u, p) \in Z \times X$ is nonsingular since $H_{u u}(\tilde{x}(t), p(t), 1)=-1(t \in T)$. Now, $\mathcal{E}(x, u, p)$ is given by those $(y, v, q) \in Z \times X$ satisfying $\dot{y}(t)=\varphi(t) v(t), \dot{q}(t)=0, \varphi(t) q(t)=v(t)$ $(t \in T)$. Let $y(t):=\int_{t}^{2}-\varphi^{2}(\tau) d \tau, v(t):=\varphi(t), q(t):=1(t \in T)$. Then $(y, v, q) \in \mathcal{E}(x, u, p),(y, v, q) \not \equiv 0, y(2)=0$ and $y(s)=0$ for all $s \in[1,2)$. Thus $[1,2) \subset \mathcal{C}(x, u, p)$. On the other hand, $\left(x_{0}, u_{0}, p\right) \equiv(0,0,0)$ belongs to $\mathcal{E}$ and $\left(x_{0}, u_{0}\right)$ solves the problem but, as we have just shown, the open interval $(0,2)$ contains "conjugate points" to 2 on $\left(x_{0}, u_{0}, p\right)$.

5.4 Example: Minimize $I(x, u)=\frac{1}{2} \int_{0}^{2 \pi}\left\{u^{2}(t)-x^{2}(t)\right\} d t$ subject to $\dot{x}(t)=\varphi(t) u(t)(t \in[0,2 \pi]), x(0)=x(2 \pi)=0$, where $\varphi(t)=0$ for $t \in[0, \pi)$ and $\varphi(t)=1$ for $t \in[\pi, 2 \pi]$.

In this case, $T=[0,2 \pi], \xi_{0}=\xi_{1}=0, \mathcal{A}=T \times \mathbf{R}^{2}, f(t, x, u)=\varphi(t) u$, $L(t, x, u)=\left(u^{2}-x^{2}\right) / 2$.

As one readily verifies, $\left(x_{0}, u_{0}\right) \equiv(0,0)$ solves the problem. Let $p \equiv 0$ so that $\left(x_{0}, u_{0}, p\right) \in \mathcal{E}$. As in the previous example, $\left(x_{0}, u_{0}\right)$ is normal to $\mathrm{P}(\mathcal{A})$ and $\left(x_{0}, u_{0}, p\right)$ is nonsingular. Now, $\mathcal{E}\left(x_{0}, u_{0}, p\right)$ is given by those $(y, v, q) \in$ $Z \times X$ satisfying $\dot{y}(t)=\varphi(t) v(t), \dot{q}(t)=-y(t), \varphi(t) q(t)=v(t)(t \in T)$. Let $(y(t), q(t)):=(0,-1)$ for $t \in[0, \pi),(y(t), q(t)):=(\sin t, \cos t)$ for $t \in[\pi, 2 \pi)$, and $v(t):=\varphi(t) q(t)(t \in T)$. Since $(y, v, q) \in \mathcal{E}\left(x_{0}, u_{0}, p\right),(y, v, q) \not \equiv 0$ and $y(\pi)=y(2 \pi)=0$, we have $\pi \in \mathcal{C}\left(x_{0}, u_{0}, p\right)$. 
These examples clearly show that some further assumptions should be imposed on $(x, u, p) \in \mathcal{E}$, with $(x, u)$ a solution of the problem, in order to have $\mathcal{C}(x, u, p)=\emptyset$.

To understand what might be needed, let us first characterize secondary extremals for the nonsingular case, as in Proposition 3.1, in terms of a linear system. As before, the following result is obtained by a direct substitution.

5.5 Proposition: Suppose $(x, u, p) \in \mathcal{L}^{\prime}$ and $(y, q) \in X \times X$. Then the following are equivalent:

a. There exists $v \in \mathcal{U}$ such that $(y, v, q) \in \mathcal{E}(x, u, p)$.

b. $(y, q)$ satisfies the linear system

$$
\dot{y}(t)=\alpha(t) y(t)+\beta(t) q(t), \quad \dot{q}(t)=\gamma(t) y(t)-\alpha^{*}(t) q(t) \quad(t \in T)
$$

which we label (J), where

$$
\begin{gathered}
\alpha(t)=A(t)-B(t) H_{u u}^{-1}(t) H_{u x}(t) \\
\beta(t)=-B(t) H_{u u}^{-1}(t) B^{*}(t) \\
\gamma(t)=H_{x u}(t) H_{u u}^{-1}(t) H_{u x}(t)-H_{x x}(t)
\end{gathered}
$$

and $H(t)$ denotes $H(\tilde{x}(t), p(t), 1)$.

Let us now extend the definition of normality to any subinterval $S$ of $T$.

5.6 Definition: For any interval $[a, b] \subset T$ we say that $(x, u) \in Z$ is (strongly) normal to $\mathrm{P}(\mathcal{A})$ on $[a, b]$ if the equations $\dot{p}(t)+A^{*}(t) p(t)=0$, $B^{*}(t) p(t)=0$ have no nonnull solution $p$ on $[a, b]$. The set of all $(x, u) \in Z$ which are normal to $\mathrm{P}(\mathcal{A})$ on $[a, b]$ will be denoted by $\mathcal{N}[a, b]$. We shall continue saying that " $(x, u)$ is normal to $\mathrm{P}(\mathcal{A})$ " if $(x, u)$ is normal to $\mathrm{P}(\mathcal{A})$ on $T$.

We now make a simple observation which helps to understand what lies behind the main result on different notions of "conjugate points" given not only in [30] but also in [28], [31] and [33].

5.7 Proposition: Suppose $s \in\left(t_{0}, t_{1}\right)$ and $(x, u, p),(y, v, q)$ in $Z \times X$ are such that

i. $(x, u, p) \in \mathcal{H} \cap \mathcal{L}^{\prime}$ with $(x, u)$ normal to $\mathrm{P}(\mathcal{A})$.

ii. $(y, v, q) \in \mathcal{E}(x, u, p)$ with $y(s)=y\left(t_{1}\right)=0$.

Then the following holds:

a. If $(x, u) \in \mathcal{N}\left[t_{0}, s\right]$ then $(y(t), v(t))=(0,0)$ for all $t \in\left[s, t_{1}\right]$.

b. If $(x, u) \in \mathcal{N}\left[s, t_{1}\right]$ then $(y(t), v(t))=(0,0)$ for all $t \in\left[t_{0}, s\right]$.

c. If $(x, u) \in \mathcal{N}\left[t_{0}, s\right] \cap \mathcal{N}\left[s, t_{1}\right]$, then $(y, v, q) \equiv(0,0,0)$ on $T$.

Proof: Let $(z(t), w(t)):=(0,0)$ if $t \in\left[t_{0}, s\right]$ and $(z(t), w(t)):=(y(t), v(t))$ if $t \in\left(s, t_{1}\right]$. Clearly

$$
J_{p}((x, u) ;(z, w))=\int_{s}^{t_{1}}\left\{\left\langle y(t), \dot{q}(t)+A^{*}(t) q(t)\right\rangle+\left\langle v(t), B^{*}(t) q(t)\right\rangle\right\} d t=0
$$


and so $(z, w)$ solves $(\mathrm{AP})$. Since $(x, u)$ is normal to $\mathrm{P}(\mathcal{A})$, so is $(z, w)$ to $(\mathrm{AP})$. By Theorem 5.1, there exists a unique $r \in X$ such that $(z, w, r) \in \mathcal{E}(x, u, p)$. In particular, this implies that

$$
\begin{gathered}
\dot{r}(t)+A^{*}(t) r(t)=0, \quad B^{*}(t) r(t)=0 \quad\left(t \in\left[t_{0}, s\right]\right) \\
{[\dot{q}(t)-\dot{r}(t)]+A^{*}(t)[q(t)-r(t)]=0, \quad B^{*}(t)[q(t)-r(t)]=0 \quad\left(t \in\left[s, t_{1}\right]\right) .}
\end{gathered}
$$

(a): Suppose $(x, u) \in \mathcal{N}\left[t_{0}, s\right]$. By $(1), r(t)=0$ for all $t \in\left[t_{0}, s\right]$. Hence $(z, r)$ satisfies $(\mathrm{J})$ with, in particular, $\left(z\left(t_{0}\right), r\left(t_{0}\right)\right)=(0,0)$, and so $(z, r) \equiv$ $(0,0)$ on $T$. Thus $y(t)=0$ for all $t \in\left[s, t_{1}\right]$. This implies that $B(t) v(t)=0$ and $B^{*}(t) q(t)=-H_{u u}(t) v(t)\left(t \in\left[s, t_{1}\right]\right)$ and, therefore,

$$
0=\left\langle v(t), B^{*}(t) q(t)\right\rangle=-\left\langle v(t), H_{u u}(t) v(t)\right\rangle \quad\left(t \in\left[s, t_{1}\right]\right) .
$$

Since $(x, u, p) \in \mathcal{L}^{\prime}$ we have $v(t)=0$ on $\left[s, t_{1}\right]$.

(b): Suppose $(x, u) \in \mathcal{N}\left[s, t_{1}\right]$. By $(2), q(t)=r(t)$ for all $t \in\left[s, t_{1}\right]$, and so we have two solutions $(y, q)$ and $(z, r)$ of $(\mathrm{J})$ satisfying, in particular, $\left(y\left(t_{1}\right), q\left(t_{1}\right)\right)=\left(z\left(t_{1}\right), r\left(t_{1}\right)\right)$. This implies that $(y, q) \equiv(z, r)$ on $T$, and so $y(t)=0$ for all $t \in\left[t_{0}, s\right]$. Proceeding as above with $\left[s, t_{1}\right]$ replaced by $\left[t_{0}, s\right]$, we conclude that $v(t)=0$ on $\left[t_{0}, s\right]$.

(c): Suppose $(x, u) \in \mathcal{N}\left[t_{0}, s\right] \cap \mathcal{N}\left[s, t_{1}\right]$. By (a) and (b), $(y, v) \equiv(0,0)$ on $T$. Therefore $\dot{q}(t)+A^{*}(t) q(t)=0$ and $B^{*}(t) q(t)=0(t \in T)$. The normality of $(x, u)$ on $T$ implies that also $q \equiv 0$.

We are now in a position to clearly understand the main theorem of [30]. With our notation, Theorem 6.1 of this paper (when applied to our problem) corresponds to the following result. The proof follows straightforwardly from Proposition 5.7c.

5.8 Theorem: Suppose $(x, u, p) \in \mathcal{E} \cap \mathcal{L}^{\prime}$ and $(x, u)$ solves $\mathrm{P}(\mathcal{A})$. If $(x, u) \in \mathcal{N}\left[a, t_{1}\right] \cap \mathcal{N}\left[t_{0}, b\right]$ for all $(a, b) \in\left[t_{0}, t_{1}\right) \times\left(t_{0}, t_{1}\right)$, then $\mathcal{C}(x, u, p) \cap$ $\left(t_{0}, t_{1}\right)=\emptyset$.

Note that, in Example 5.3, any $(x, u) \in Z$ satisfies $(x, u) \in \mathcal{N}[a, 2] \cap$ $\mathcal{N}[0, b]$ for all $(a, b) \in[0,1) \times(0,2)$, but $(x, u) \notin \mathcal{N}[a, 2]$ if $a \in[1,2)$. In Example 5.4, any $(x, u) \in Z$ satisfies $(x, u) \in \mathcal{N}[a, 2 \pi] \cap \mathcal{N}[0, b]$ for all $(a, b) \in$ $[0,2 \pi) \times[\pi, 2 \pi)$, but $(x, u) \notin \mathcal{N}[0, b]$ if $b \in(0, \pi)$.

In view of this fact, according to Remark 6.1 of [30], these examples "illustrate that a one-sided strong normality in the theorem is not enough for the result to hold true. In other words, the strong normality assumption on either side is indispensable and cannot be weakened."

In the introduction of [17] one reads: "The claim of Zeidan and Zezza that the normality conditions on both ends are indispensable is because their definition of conjugate points [...] forces them to impose this condition." 
This is clearly deduced from Proposition 5.7. In the definition given in [30], the corresponding $(y, v, q) \in \mathcal{E}(x, u, p)$ with $y(s)=y\left(t_{1}\right)=0$ should satisfy $(y, v, q) \not \equiv(0,0,0)$ on $T$. But there is no need to impose such a strong condition. By $5.7 \mathrm{a}$, it suffices to have $y \not \equiv 0$ on $\left[s, t_{1}\right]$. Then the nonexistence of such points in the open interval follows, under the first assumptions of Theorem 5.8, if $(x, u) \in \mathcal{N}\left[t_{0}, b\right]$ for all $b \in\left(t_{0}, t_{1}\right]$. The same occurs, by $5.7 \mathrm{~b}$, if one imposes the condition $y \not \equiv 0$ on $\left[t_{0}, s\right]$ and $(x, u) \in \mathcal{N}\left[a, t_{1}\right]$ for all $a \in\left[t_{0}, t_{1}\right)$. Let us formalize these facts.

5.9 Definition: For any $(x, u, p) \in Z \times X$ let $\mathcal{C}_{0}(x, u, p)\left(\operatorname{resp} \mathcal{C}_{1}(x, u, p)\right)$ be the set of points $s \in\left[t_{0}, t_{1}\right)$ for which there exists $(y, v, q) \in \mathcal{E}(x, u, p)$ with $y(s)=y\left(t_{1}\right)=0$ and $y \not \equiv 0$ on $\left[t_{0}, s\right]\left(\operatorname{resp}\left[s, t_{1}\right]\right)$.

One readily verifies that, under the assumptions of Theorem $5.8, \mathcal{C}(x, u, p) \subset$ $\mathcal{C}_{0}(x, u, p) \cup \mathcal{C}_{1}(x, u, p)$. Hence, this theorem is a corollary of the following result.

5.10 Theorem: Suppose $(x, u, p) \in \mathcal{E} \cap \mathcal{L}^{\prime}$ and $(x, u)$ solves $\mathrm{P}(\mathcal{A})$. Then the following holds:

a. $(x, u) \in \mathcal{N}\left[a, t_{1}\right]$ for all $a \in\left[t_{0}, t_{1}\right) \Rightarrow \mathcal{C}_{0}(x, u, p) \cap\left(t_{0}, t_{1}\right)=\emptyset$.

b. $(x, u) \in \mathcal{N}\left[t_{0}, b\right]$ for all $b \in\left(t_{0}, t_{1}\right] \Rightarrow \mathcal{C}_{1}(x, u, p) \cap\left(t_{0}, t_{1}\right)=\emptyset$.

Let us make a few comments on subsequent papers of Zeidan and Zezza.

- In [31], second order conditions are derived from [13] or [27] upon writing the problem in Mayer form, and they follow an approach similar to that of [30]. An example is given "which illustrates the indispensability of the normality assumption on either side."

- In [33], they consider the problem of [31] with a general boundary condition and $U$ open. Second order conditions are again derived from [13] or [27]. In this paper, they make one of the modifications of $\mathcal{C}(x, u, p)$ explained above. Their definition of "points coupled with $t_{1}$ " is based explicitly on the linear system $(\mathrm{J})$ (see Proposition 5.5) assuming that $(x, u, p) \in \mathcal{E} \cap \mathcal{L}^{\prime}$. Then, for a point $s \in\left[t_{0}, t_{1}\right)$, it is asked the existence of $(y, q) \in X \times X$ satisfying $(\mathrm{J})$ with $y(s)=y\left(t_{1}\right)=0$ and

$$
v(t):=-H_{u u}^{-1}(\tilde{x}(t), p(t), 1)\left[H_{u x}(\tilde{x}(t), p(t), 1) y(t)+B^{*}(t) q(t)\right] \not \equiv 0 \text { on }\left[t_{0}, s\right] .
$$

This modification of their previous definition of conjugacy correspons to $\mathcal{C}_{0}(x, u, p)$, and their main result (applied to our problem) is precisely Theorem 5.10a.

- The problem considered in [28] is that of [31] with $\mathcal{U}=L^{\infty}\left(T, \mathbf{R}^{m}\right)$, $O=\mathbf{R}^{n}, V=\mathbf{R}^{m}$, and

$$
\mathcal{A}=\left\{(t, x, u) \in T \times \mathbf{R}^{n} \times \mathbf{R}^{m} \mid \varphi(t, x(t), u(t)) \leq 0(t \in T)\right\} .
$$

Second order conditions are quoted from [20], and conjugacy is defined as in 5.9 in terms of $\mathcal{C}_{0}(x, u, p)$. There, a point belonging to $\mathcal{C}(x, u, p)$ is called 
"classically conjugate to $t_{1}$ along $(x, u)$." Again, the main theorem (applied to our problem) corresponds to Theorem 5.10a.

Let us now generalize, for problem $\mathrm{P}(\mathcal{A})$, the set $\mathcal{P}$ introduced in Section 3.

Let us begin with the following notation. For any $s \in\left[t_{0}, t_{1}\right)$ denote by $X_{s}$ the space of piecewise $C^{1}$ functions mapping $\left[s, t_{1}\right]$ to $\mathbf{R}^{n}$, by $\mathcal{U}_{s}$ the space of piecewise continuous functions mapping $\left[s, t_{1}\right]$ to $\mathbf{R}^{m}$, set $Z_{s}:=X_{s} \times \mathcal{U}_{s}$, and let $\mathcal{E}_{s}(x, u, p)$ be the set of all $(y, v, q) \in Z_{s} \times X_{s}$ satisfying, for all $t \in\left[s, t_{1}\right]$,

$$
\begin{gathered}
\dot{y}(t)=A(t) y(t)+B(t) v(t) \\
\dot{q}(t)+A^{*}(t) q(t)=-H_{x x}(\tilde{x}(t), p(t), 1) y(t)-H_{x u}(\tilde{x}(t), p(t), 1) v(t) \\
B^{*}(t) q(t)=-H_{u x}(\tilde{x}(t), p(t), 1) y(t)-H_{u u}(\tilde{x}(t), p(t), 1) v(t) .
\end{gathered}
$$

5.11 Definition: Given $(x, u, p) \in Z \times X$ let $\mathcal{P}_{1}(x, u, p)$ be the set of points $s \in\left[t_{0}, t_{1}\right)$ for which there exist $(y, v, q) \in Z_{s} \times X_{s}$ and $\epsilon>0$ such that

i. If $s>t_{0}$ then $(x, u) \in \mathcal{N}([s-\epsilon, s] \cap T)$.

ii. $(y, v, q) \in \mathcal{E}_{s}(x, u, p)$ with $y(s)=y\left(t_{1}\right)=0$ and $y \not \equiv 0$ on $[s, s+\epsilon] \cap T$.

iii. $H_{u u}(\tilde{x}(t), p(t), 1)<0$ for all $t \in[s-\epsilon, s+\epsilon] \cap T$.

Note that, under the assumptions of Theorem 5.10b, $\mathcal{C}_{1}(x, u, p) \subset \mathcal{P}_{1}(x, u, p)$ (this follows simply by choosing $\epsilon=\max \left\{s-t_{0}, t_{1}-s\right\}$ ). Also, we can proceed in a similar way with respect to $\mathcal{C}_{0}(x, u, p)$, defining a set $\mathcal{P}_{0}(x, u, p)$, by considering functions in $\mathcal{E}_{s-\epsilon}(x, u, p)$ and making the obvious modifications in the definition of $\mathcal{P}_{1}(x, u, p)$. For simplicity, we shall only deal with $\mathcal{P}(x, u, p):=\mathcal{P}_{1}(x, u, p)$.

5.12 Theorem: If $(x, u, p) \in \mathcal{H}$ with $(x, u) \in Z(\mathcal{A})$ normal to $\mathrm{P}(\mathcal{A})$ then $\mathcal{P}(x, u, p) \cap\left(t_{0}, t_{1}\right)=\emptyset$.

Proof: We proceed basically as in 5.7a. Suppose there exists $s \in \mathcal{P}(x, u, p) \cap$ $\left(t_{0}, t_{1}\right)$. Let $(y, v, q) \in Z_{s} \times X_{s}$ and $\epsilon>0$ be as in 5.11. Since $s \in\left(t_{0}, t_{1}\right)$ we can suppose, without loss of generality, that $\epsilon<\min \left\{s-t_{0}, t_{1}-s\right\}$. Define $(z(t), w(t)):=(0,0)$ if $t \in\left[t_{0}, s\right]$ and $(z(t), w(t)):=(y(t), v(t))$ if $t \in\left(s, t_{1}\right]$. Then $(z, w)$ solves $(\mathrm{AP})$. Since $(x, u)$ is normal to $\mathrm{P}(\mathcal{A})$, so is $(z, w)$ to $(\mathrm{AP})$. By Theorem 5.1, there exists a unique $r \in X$ such that $(z, w, r) \in \mathcal{E}(x, u, p)$. In particular, this implies that

$$
\dot{r}(t)+A^{*}(t) r(t)=0, \quad B^{*}(t) r(t)=0 \quad\left(t \in\left[t_{0}, s\right]\right) .
$$

Since $(x, u) \in \mathcal{N}[s-\epsilon, s], r(t)=0$ for all $t \in[s-\epsilon, s]$. Hence $(z, r)$ satisfies $(\mathrm{J})$ on $[s-\epsilon, s+\epsilon]$ with, in particular, $(z(t), r(t))=(0,0)$ for any $t \in(s-\epsilon, s)$, and so $(z, r) \equiv(0,0)$ on $[s-\epsilon, s+\epsilon]$. Thus $y(t)=0$ for all $t \in[s, s+\epsilon]$, contradicting (ii). 
We turn now to generalize the set $\mathcal{Q}$ of Section 3. For this set, its emptiness in the open time interval is necessary for normal minimizers without even one-sided normality assumptions.

5.13 Definition: Given $(x, u, p) \in Z \times X$ let $\mathcal{Q}(x, u, p)$ be the set of points $s \in\left[t_{0}, t_{1}\right)$ for which there exists $(y, v, q) \in \mathcal{E}_{s}(x, u, p)$ with $y(s)=$ $y\left(t_{1}\right)=0, H_{u u}(\tilde{x}(s), p(s), 1) v(s) \neq 0$, and $B$ is continuous at $s$.

5.14 Theorem: If $(x, u, p) \in \mathcal{H}$ with $(x, u) \in Z(\mathcal{A})$ normal to $\mathrm{P}(\mathcal{A})$ then $\mathcal{Q}(x, u, p) \cap\left(t_{0}, t_{1}\right)=\emptyset$.

Proof: Suppose there exists $s \in \mathcal{Q}(x, u, p) \cap\left(t_{0}, t_{1}\right)$. Let $(y, v, q) \in$ $\mathcal{E}_{s}(x, u, p)$ be as in 5.13. Define $(z(t), w(t)):=(0,0)$ if $t \in\left[t_{0}, s\right]$ and $(z(t), w(t)):=$ $(y(t), v(t))$ if $t \in\left(s, t_{1}\right]$. Then $(z, w)$ solves (AP) and, since $(x, u)$ is normal to $\mathrm{P}(\mathcal{A})$, so is $(z, w)$ to $(\mathrm{AP})$. By Theorem 5.1 , there exists a unique $r \in X$ such that $(z, w, r) \in \mathcal{E}(x, u, p)$. Note that $B^{*}(s) r(s)=-H_{u u}(\tilde{x}(s), p(s), 1) w(s)$ and the function $t \mapsto B^{*}(t) r(t)$ is continuous at $s$. But $B^{*}(s-) r(s-)=0 \neq$ $B^{*}(s+) r(s+)$, and we reach a contradiction.

It might be tempting to think that the continuity of $B$ at $s$, in the definition of $\mathcal{Q}(x, u, p)$, is redundant or unnecessary. Indeed, following the proof up to " $(z, w, r) \in \mathcal{E}(x, u, p)$," the function

$\psi(t):=\tilde{H}(t, z(t), w(t), r(t), 1)=\langle r(t), A(t) z(t)+B(t) w(t)\rangle-\Omega(t, z(t), w(t))(t \in T)$

is continuous if, with respect to the accessory problem, assumption (B) holds (see Note 4.3). However, under our assumptions on $L$ and $f$ (see (A1)), $\psi$ may be discontinuous. Example 5.4 clearly illustrates this fact.

The following examples, which are simple modifications of Example 5.4, illustrate the usefulness of the sets just introduced. We shall compare the applicability of Theorems 5.8 and 5.10 with that of 5.12 or 5.14.

5.15 Example: Consider the problem of minimizing

$$
I(x, u)=\frac{1}{2} \int_{-\pi}^{\pi}\left\{u^{2}(t)-x^{2}(t)\right\} d t
$$

subject to $\dot{x}(t)=\varphi(t) u(t)(t \in[-\pi, \pi])$ and $x(-\pi)=x(\pi)=0$, where

$$
\varphi(t)= \begin{cases}0 & \text { if } t \in[-\pi,-1] \\ h(t) & \text { if } t \in[-1,0] \\ 1 & \text { if } t \in[0, \pi]\end{cases}
$$

and $h:[-1,0] \rightarrow \mathbf{R}$ is any positive, continuous function.

In this case we have $T=[-\pi, \pi], n=m=1, \xi_{0}=\xi_{1}=0, \mathcal{A}=T \times \mathbf{R}^{2}$, $L(t, x, u)=\frac{1}{2}\left(u^{2}-x^{2}\right) \quad$ and $\quad f(t, x, u)=\varphi(t) u \quad((t, x, u) \in \mathcal{A})$. 
Let $(x, u) \in Z$. Note that $(x, u)$ is normal to $\mathrm{P}(\mathcal{A})$ on $[a, b]$ if the equations $\dot{p}(t)=0, \varphi(t) p(t)=0$ have no nonnull solution $p$ on $[a, b]$. Thus $(x, u) \in \mathcal{N}[a, \pi]$ for all $a \in[-\pi, \pi)$ but $(x, u) \notin \mathcal{N}[-\pi, b]$ if $b \in(-\pi,-1]$. Therefore, we cannot apply Theorems 5.8 or $5.10 \mathrm{~b}$. Note that $\mathcal{E}(x, u, p)$ is given by those $(y, v, q) \in Z \times X$ satisfying $\dot{y}(t)=\varphi(t) v(t), \dot{q}(t)=-y(t)$, $\varphi(t) q(t)=v(t)(t \in T)$. If we try to apply Theorem 5.10a, we need to find $s \in(-\pi, \pi)$ and $(y, v, q) \in \mathcal{E}(x, u, p)$ with $y(s)=y(\pi)=0$ and $y \not \equiv 0$ on $[-\pi, s]$. Note that, in particular, we require

$$
\dot{q}(t)=-y(t)(t \in[-\pi, \pi]) \quad \text { and } \quad \dot{y}(t)= \begin{cases}0 & \text { if } t \in[-\pi,-1] \\ h^{2}(t) q(t) & \text { if } t \in[-1,0] \\ q(t) & \text { if } t \in[0, \pi]\end{cases}
$$

Let us proceed with Theorems 5.12 and 5.14. Let $(y(t), v(t), q(t)):=(\sin t, \cos t, \cos t)$ for all $t \in[0, \pi]$. Then $(y, v, q) \in \mathcal{E}_{s}(x, u, p)$ with $s=0$. Let $0<\epsilon \leq \pi$ (note that we can choose $\epsilon=\pi)$. Since $(x, u) \in \mathcal{N}[-\epsilon, 0], y \not \equiv 0$ on $[0, \epsilon]$ and $H_{u u}(\tilde{x}(t), p(t), 1)=-1$ for all $t \in[-\epsilon, \epsilon]$, we have $0 \in \mathcal{P}(x, u, p)$. By Theorem 5.12 , the problem has no solution. Note that the same conclusion follows by Theorem 5.14 if $\varphi$ is continuous at 0 (that is, if $h(0)=1$ ) since, in this case, $0 \in \mathcal{Q}(x, u, p)$.

5.16 Example: Consider the problem of minimizing

$$
I(x, u)=\frac{1}{2} \int_{-\pi}^{\pi}\left\{\varphi(t) u^{2}(t)-x^{2}(t)\right\} d t
$$

subject to $\dot{x}(t)=\varphi(t) u(t)(t \in[-\pi, \pi])$ and $x(-\pi)=x(\pi)=0$, where $\varphi$ is as in Example 5.15.

In this case we have $T=[-\pi, \pi], n=m=1, \xi_{0}=\xi_{1}=0, \mathcal{A}=T \times \mathbf{R}^{2}$,

$$
L(t, x, u)=\frac{1}{2}\left(\varphi(t) u^{2}-x^{2}\right) \quad \text { and } \quad f(t, x, u)=\varphi(t) u \quad((t, x, u) \in \mathcal{A}) .
$$

Observe first that any $(x, u) \in Z$ is normal to $\mathrm{P}(\mathcal{A})$. Also, $H_{u u}(\tilde{x}(t), p(t), 1)=$ $-\varphi(t)$ for any $(x, u, p) \in Z \times X$ and so $\mathcal{L}^{\prime}=\emptyset$ (any $(x, u, p)$ is singular). Therefore, we cannot apply Theorems 5.8 or 5.10. Now, $\mathcal{E}_{s}(x, u, p)$ is given by those $(y, v, q) \in Z_{s} \times X_{s}$ satisfying $\dot{y}(t)=\varphi(t) v(t), \dot{q}(t)=-y(t), \varphi(t) q(t)=\varphi(t) v(t)$ $\left(t \in\left[s, t_{1}\right]\right)$. Thus, the function $(y(t), v(t), q(t))=(\sin t, \cos t, \cos t)(t \in[0, \pi])$ shows, as in the previous example, that $0 \in \mathcal{P}(x, u, p)$ (or, if $h(0)=1$, $0 \in \mathcal{Q}(x, u, p)$ ). By Theorem 5.12 (or 5.14 if $h(0)=1$ ), this problem has no solution.

We end this section with a brief remark related to the sets $\mathcal{E}(x)$ and $\mathcal{J}(x)$ introduced in Section 3 (see the note before Definition 5.2). In the definitions of conjugacy given in this section, we can replace $\mathcal{E}_{s}(x, u, p)$ with 
a set $\mathcal{J}_{s}(x, u, p)$, as in the calculus of variations problem, defined exactly as $\mathcal{E}_{s}(x, u, p)$ but without imposing the condition that the function $q$ should belong to $X_{s}$. In that event, the proof of Proposition 5.7 remains with no changes since the function $(z, w)$ in that proof again minimizes the accessory problem. As before, we would have $\mathcal{E}_{s}(x, u, p) \subset \mathcal{J}_{s}(x, u, p)$ and this contention may be proper. A simple variant of Example 5.15 illustrates some of the advantages of this modification.

5.17 Example: Consider the problem of minimizing

$$
I(x, u)=\frac{1}{2} \int_{-\pi}^{2 \pi}\left\{u^{2}(t)-x^{2}(t)\right\} d t
$$

subject to $\dot{x}(t)=\varphi(t) u(t)(t \in[-\pi, 2 \pi])$ and $x(-\pi)=x(2 \pi)=0$, where

$$
\varphi(t)= \begin{cases}0 & \text { if } t \in[-\pi,-1] \cup[\pi+1,2 \pi] \\ t+1 & \text { if } t \in[-1,0] \\ 1 & \text { if } t \in[0, \pi] \\ \pi+1-t & \text { if } t \in[\pi, \pi+1]\end{cases}
$$

In this case we have $T=[-\pi, 2 \pi], n=m=1, \xi_{0}=\xi_{1}=0, \mathcal{A}=T \times \mathbf{R}^{2}$,

$$
L(t, x, u)=\frac{1}{2}\left(u^{2}-x^{2}\right) \quad \text { and } \quad f(t, x, u)=\varphi(t) u \quad((t, x, u) \in \mathcal{A}) .
$$

Note that, though any $(x, u) \in Z$ is normal to $\mathrm{P}(\mathcal{A})$ (on the entire interval $[-\pi, 2 \pi])$, none of the Theorems 5.8 or 5.10 can be applied since $(x, u) \notin \mathcal{N}[-\pi, b]$ if $b \in(-\pi,-1]$ and $(x, u) \notin \mathcal{N}[a, 2 \pi]$ if $a \in[\pi+1,2 \pi)$. Let $(y(t), v(t), q(t)):=(\sin t, \cos t, \cos t)$ for all $t \in[0, \pi]$. If we set $(y(t), v(t), q(t)):=$ $(0,0,0)$ for all $t \in(\pi, 2 \pi]$, then $(y, v, q) \notin \mathcal{E}_{0}(x, u, p)$ since $q$ is not continuous at $\pi$. However, $(y, v, q) \in \mathcal{J}_{0}(x, u, p)$ and, proceeding as in Example 5.15 (with $\mathcal{E}_{s}(x, u, p)$ replaced by $\mathcal{J}_{s}(x, u, p)$ in Definitions 5.11 and 5.13$)$, we conclude that $0 \in \mathcal{P}(x, u, p)$ (and $0 \in \mathcal{Q}(x, u, p)$ ). Thus, $\mathrm{P}(\mathcal{A})$ has no solution.

\section{Conjugate intervals}

So far we have dealt with two approaches to conjugacy, both of which require the existence of a nonzero "secondary extremal" $(y, v, q)$ with $y$ vanishing at the conjugate points. An application of the maximum principle to a solution $(z, w)$ of the accessory problem, obtained by extending $(y, v)$ to zero outside the interval containing the two points, yields the existence of some piecewise $C^{1}$ function $r$. One of the approaches relies on the uniqueness of solutions of the linear system $(\mathrm{J})$ which is satisfied (at least locally) by $(z, w, r)$. The other depends basically on the continuity of $r$. 


\section{Loewen and Zheng}

An entirely different approach was devised by Loewen and Zheng [17] in 1994. The optimal control problem they consider is that of [30] (see the statement of the problem in Section 5) but involving equality and inequality constraints in the control, that is, $\mathcal{A}=T \times O \times U$ where

$$
U=\left\{u \in V \mid \varphi_{\alpha}(u)=0(\alpha \in A), \varphi_{\beta}(u) \leq 0(\beta \in B)\right\}
$$

with $A, B$ disjoint index sets. Second order conditions are derived assuming convexity of $U$. Their approach is then based, essentially, on a set of "generalized conjugate points" whose nonemptiness implies the existence of a negative second variation.

In [30, Important Remark 6.4], Zeidan and Zezza claim that the inequality constrained case is within the scope of their paper. However, according to [17], "Their justification is neither clear nor convincing [...] because control sets defined by a mixed constraint system may have corners, whereas the methods of [30] are valid exclusively for smooth control sets." We shall not discuss whose claim is correct. Let us just point out that the presence of this kind of constraints is a crucial feature in the theory developed in [17].

Another fundamental aspect in [17] is their approach to conjugacy, and we shall concentrate on it. Throughout the paper, the authors compare their results with those of [30] (and occasionally with [31]). In particular, they state that "when the strengthened Legendre condition and the strong normality conditions of [30] fail to hold, the results of [30] and [31] give little information." "Some examples will show how our generalized conjugate point gives much more information than that of [30]," and "our generalized conjugate point is sharper and more general than that of [31]."

In order to clearly understand the approach given in [17], let us return to problem $\mathrm{P}(\mathcal{A})$ of Section 4. The assumptions of [17] yielding first and second order conditions are stronger than those of [30] (in particular $f$ and $L$ are assumed to be of class $C^{2}$ ), and so we shall continue assuming that (A1) and (A2) (as modified in Section 3) hold.

Let us first introduce the following notation. For all $s \in\left[t_{0}, t_{1}\right)$ let $T_{s}:=\left[s, t_{1}\right]$, and recall that $X_{s}$ denotes the space of piecewise $C^{1}$ functions mapping $T_{s}$ to $\mathbf{R}^{n}$, and $\mathcal{U}_{s}$ the space of piecewise continuous functions mapping $T_{s}$ to $\mathbf{R}^{m}$. Now let

$Y_{s}(x, u):=\left\{(y, v) \in X_{s} \times \mathcal{U}_{s} \mid \dot{y}(t)=A(t) y(t)+B(t) v(t)\left(t \in T_{s}\right), y(s)=y\left(t_{1}\right)=0\right\}$

where $A(t)=f_{x}(\tilde{x}(t)), B(t)=f_{u}(\tilde{x}(t))(t \in T)$. Whenever we are given $(x, u, p) \in Z \times X$ and $(y, v) \in Y_{s}(x, u)$, we shall consider the functions $\sigma: T_{s} \rightarrow$ $\mathbf{R}^{n}$ and $\rho: T_{s} \rightarrow \mathbf{R}^{m}$ defined by

$$
\sigma(t):=-H_{x x}(t) y(t)-H_{x u}(t) v(t), \quad \rho(t):=-H_{u x}(t) y(t)-H_{u u}(t) v(t)
$$


where $H(t)$ denotes $H(\tilde{x}(t), p(t), 1)$.

Let us now introduce, for problem $\mathrm{P}(\mathcal{A})$, the set of "generalized conjugate points" defined in [17].

6.1 Definition: For any $(x, u, p) \in Z \times X$ let $\mathcal{G}_{1}(x, u, p)$ be the set of points $s \in\left[t_{0}, t_{1}\right)$ for which there exist $(y, v) \in Y_{s}(x, u)$ and $q \in X_{s}$ such that, if $\lambda(t):=B^{*}(t) q(t)-\rho(t)\left(t \in T_{s}\right)$, then

i. $\dot{q}(t)+A^{*}(t) q(t)=\sigma(t)\left(t \in T_{s}\right)$.

ii. $q(s) \neq 0$.

iii. $\langle v(t), \lambda(t)\rangle \geq 0\left(t \in T_{s}\right)$

and either (a) or (b) holds:

a. $\langle v(t), \lambda(t)\rangle>0$ on a set of positive measure.

b. There exists $(z, w) \in Y(x, u)$ such that

i. $\langle z(s), q(s)\rangle>0$.

ii. $\langle w(t), \lambda(t)\rangle \geq 0\left(t \in T_{s}\right)$.

The main result in $[17$, Theorem 4.3] relating this set to the condition " $(x, u, p) \in \mathcal{H} "$ is the following.

6.2 Theorem: Suppose $(x, u, p) \in \mathcal{E}$. Then $(x, u, p) \in \mathcal{H} \Rightarrow \mathcal{G}_{1}(x, u, p) \cap$ $\left(t_{0}, t_{1}\right)=\emptyset$.

This result and Theorem 5.1 yield the following necessary condition for optimality: if $(x, u, p) \in \mathcal{E}$, with $(x, u)$ a normal solution to $\mathrm{P}(\mathcal{A})$, then the open interval $\left(t_{0}, t_{1}\right)$ contains no points in $\mathcal{G}_{1}(x, u, p)$.

Note that this notion of conjugacy does not generalize the classical one of [14] since the nonexistence of such points in $\left(t_{0}, t_{1}\right)$ is necessary for optimality even if the process under consideration is singular. However, if the problem considered in [17] is reduced to the calculus of variations problem and the extremal under consideration is nonsingular, then the classical set $\mathcal{C}(x)$ of conjugate points, in the open time interval, is contained in that of generalized conjugate points. A simple proof of this fact is given in [17].

Thus this new notion "extends" that of conjugacy not only for optimal control problems but also in the classical theory of calculus of variations. Several examples given in [17] illustrate the usefulness of this set. They deal with singular processes, so that the classical theory of [14], or that of [30], fail to detect nonoptimality, while this fact follows by proving the existence of generalized conjugate points.

Though the introduction of this set seems to be successful for certain quite general classes of optimal control problems, it presents two undesirable features. First, its nonemptiness has been established merely as a sufficient condition for the existence of negative second variations. In other words, it is not clear if there are problems with negative second variations for which 
this set is empty. Second, one should bear in mind that the main objective of introducing a characterization of a second order condition should be, of course, to obtain a simpler way of verifying it. However, even for simple problems in the calculus of variations, one can easily find examples for which to solve the question of nonemptiness of this set may be much more difficult than verifying directly if that condition holds (see [2], [3], [4]).

With the purpose of solving these two problems, we introduced in [2] a set of points, say $\mathcal{R}(x)$, applicable to the calculus of variations problem. For this set, $x \in \mathcal{H}$ if and only if $\mathcal{R}(x)$ is empty. Further, this set enjoys the property that, if there exists an admissible variation $y$ for which the second variation is negative, then $y$ trivially satisfies the conditions defining membership of $\mathcal{R}(x)$. In other words, it can never be more difficult to check nonemptiness of $\mathcal{R}(x)$ than to prove directly that $x \notin \mathcal{H}$. Finally, we proved in [4] that $\mathcal{G}_{1}(x, u, p)$ (in the calculus of variations context) is contained in $\mathcal{R}(x)$. Let us generalize this set to $\mathrm{P}(\mathcal{A})$.

6.3 Definition: For any $(x, u, p) \in Z \times X$ let $\mathcal{R}_{1}(x, u, p)$ be the set of points $s \in\left[t_{0}, t_{1}\right)$ for which there exists $(y, v) \in Y_{s}(x, u)$ such that

i. $\int_{s}^{t_{1}}\{\langle y(t), \sigma(t)\rangle+\langle v(t), \rho(t)\rangle\} d t \leq 0$.

ii. There exists $(z, w) \in Y(x, u)$ such that $\gamma:=$ $\int_{s}^{t_{1}}\{\langle z(t), \sigma(t)\rangle+\langle w(t), \rho(t)\rangle\} d t \neq 0$.

6.4 Theorem: Let $(x, u, p) \in Z \times X$. Then the following holds:

a. $\mathcal{G}_{1}(x, u, p) \subset \mathcal{R}_{1}(x, u, p)$.

b. $(x, u, p) \in \mathcal{H} \Leftrightarrow \mathcal{R}_{1}(x, u, p)=\emptyset$.

Proof:

(a): Let $s \in \mathcal{G}_{1}(x, u, p)$ and let $(y, v) \in Y_{s}(x, u)$ and $q \in X_{s}$ be as in 6.1. Condition $6.3(\mathrm{i})$ is a consequence of 6.1 (iii) since

$$
\begin{gathered}
\int_{s}^{t_{1}}\{\langle y(t), \sigma(t)\rangle+\langle v(t), \rho(t)\rangle\} d t= \\
\int_{s}^{t_{1}}\left\{\left\langle y(t), \dot{q}(t)+A^{*}(t) q(t)\right\rangle+\left\langle v(t), B^{*}(t) q(t)-\lambda(t)\right\rangle\right\} d t \\
=\int_{s}^{t_{1}}\{\langle y(t), \dot{q}(t)\rangle+\langle A(t) y(t)+B(t) v(t), q(t)\rangle-\langle v(t), \lambda(t)\rangle\} d t \\
=-\int_{s}^{t_{1}}\langle v(t), \lambda(t)\rangle d t \leq 0 .
\end{gathered}
$$

If $6.1 \mathrm{a}$ holds, then the inequality in $6.3(\mathrm{i})$ is strict and so $s \in \mathcal{R}_{1}(x, u, p)$. If $6.1 \mathrm{~b}$ holds, then there exists $(z, w) \in Y(x, u)$ such that $\langle z(s), q(s)\rangle>0$ and $\langle w(t), \lambda(t)\rangle \geq 0\left(t \in T_{s}\right)$. Therefore,

$\gamma=\int_{s}^{t_{1}}\{\langle z(t), \sigma(t)\rangle+\langle w(t), \rho(t)\rangle\} d t=-\langle z(s), q(s)\rangle-\int_{s}^{t_{1}}\langle w(t), \lambda(t)\rangle d t<0$. 
(b) " $\Rightarrow$ ": Suppose there exists $s \in \mathcal{R}_{1}(x, u, p)$. Let $(y, v)$ and $(z, w)$ be as in 6.3. Note that, since $\gamma \neq 0,(y, v) \not \equiv(0,0)$. Let $(\zeta(t), \eta(t)):=(0,0)$ if $t \in\left[t_{0}, s\right],(\zeta(t), \eta(t)):=(y(t), v(t))$ if $t \in\left(s, t_{1}\right]$, and observe that, by $6.3(\mathrm{i})$,

$$
J_{p}((x, u) ;(\zeta, \eta))=\int_{t_{0}}^{t_{1}} 2 \Omega(t, \zeta(t), \eta(t)) d t=\int_{s}^{t_{1}}\{\langle y(t), \sigma(t)\rangle+\langle v(t), \rho(t)\rangle\} d t \leq 0 .
$$

Set $k:=J_{p}((x, u) ;(z, w))$ and $\alpha:=-(\gamma+k / 2 \gamma)$. Then $\left(y_{\alpha}, v_{\alpha}\right):=(z+\alpha \zeta, w+$ $\alpha \eta)$ belongs to $Y(x, u)$ and

$$
\begin{gathered}
J_{p}\left((x, u) ;\left(y_{\alpha}, v_{\alpha}\right)\right)=\int_{t_{0}}^{t_{1}} 2 \Omega\left(t, y_{\alpha}(t), v_{\alpha}(t)\right) d t \\
=k+\alpha^{2} J_{p}((x, u) ;(\zeta, \eta))+2 \alpha \int_{s}^{t_{1}}\{\langle z(t), \sigma(t)\rangle+\langle w(t), \rho(t)\rangle\} d t \\
\leq k+2 \alpha \gamma=-2 \gamma^{2}<0 .
\end{gathered}
$$

(b) "Æ": Suppose $(x, u, p) \notin \mathcal{H}$. Let $(y, v) \in Y(x, u)$ be such that $J_{p}((x, u) ;(y, v))<0$ and let $(z, w) \equiv(y, v)$. Then $t_{0} \in \mathcal{R}_{1}(x, u, p)$.

6.5 Remark: Note that, in view of this result, $(x, u, p) \in \mathcal{H} \Rightarrow \mathcal{G}_{1}(x, u, p)=$ $\emptyset$. In other words, a necessary condition for optimality is the nonexistence of generalized conjugate points in the half-open interval $\left[t_{0}, t_{1}\right)$, and not only in $\left(t_{0}, t_{1}\right)$. Note also that, in the proof of Theorem $6.4 \mathrm{a}$, the condition " $q(s) \neq 0$ " is never used so that, in Definition 6.1, it can be removed. The reason is that, if $6.1 \mathrm{a}$ holds, then it is unnecessary and, if $6.1 \mathrm{~b}$ holds, it is redundant. Finally recall that, in the two approaches of Section 5, we require (see Definitions 5.11 and 5.13) the existence of $(y, v, q) \in \mathcal{E}_{s}(x, u, p)$ with $y(s)=y\left(t_{1}\right)=0$, that is, $(y, v) \in Y_{s}(x, u)$ and $q \in X_{s}$ satisfying

$$
\dot{q}(t)+A^{*}(t) q(t)=\sigma(t), \quad B^{*}(t) q(t)=\rho(t) \quad\left(t \in T_{s}\right) .
$$

Therefore, the definition of $\mathcal{G}_{1}(x, u, p)$ allows us to consider functions $q \in X_{s}$ for which $\lambda(t)=B^{*}(t) q(t)-\rho(t)$ may not vanish. In this sense this approach is more general than the previous ones. However observe that, in the definition of $\mathcal{R}_{1}(x, u, p)$, the function $q \in X_{s}$ is not required at all. We refer the reader to [4] for a wide range of problems for which verifying membership of $\mathcal{R}_{1}(x, u, p)$ is trivial but that of $\mathcal{G}_{1}(x, u, p)$ may be extremely difficult or perhaps even a hopless task.

\section{Zeidan}

The paper by Loewen and Zheng had an important impact in the work by Zeidan who, so far, had given possible generalizations of conjugacy from a 
classical point of view. In 1996, Zeidan [29] introduced a new set of "generalized coupled points," containing that of [17] and defined for certain classes of optimal control problems where both endpoints vary and the control set is not necessarily convex. For the normal case, it is shown that a necessary condition for optimality is again the nonexistence of such points in $\left(t_{0}, t_{1}\right)$.

When reduced to problem $\mathrm{P}(\mathcal{A})$ (and making the necessary changes since [29] refers to a minimum instead of a maximum principle), this set corresponds to the following.

6.6 Definition: For any $(x, u, p) \in Z \times X$ let $\mathcal{G}_{2}(x, u, p)$ be the set of points $s \in\left[t_{0}, t_{1}\right)$ for which there exist $(y, v) \in Y_{s}(x, u)$ and $q \in X_{s}$ such that, if $\lambda(t):=B^{*}(t) q(t)-\rho(t)\left(t \in T_{s}\right)$, then

i. $\dot{q}(t)+A^{*}(t) q(t)=\sigma(t)\left(t \in T_{s}\right)$.

ii. $\langle v(t), \lambda(t)\rangle \geq 0\left(t \in T_{s}\right)$

iii. If the inequality in (ii) is equality for all $t \in T_{s}$ then, for any $\alpha \in \mathbf{R}^{n}$ satisfying $\lambda(t)=B^{*}(t) \Phi^{*}(t) \alpha\left(t \in T_{s}\right)$, there exists $w:\left[t_{0}, s\right] \rightarrow \mathbf{R}^{m}$ piecewise continuous with $\left\langle z(s), \Phi^{*}(s) \alpha-q(s)\right\rangle<0$, where $\Phi: T \rightarrow \mathbf{R}^{n \times n}$ satisfies $\dot{\Phi}(t)=$ $-\Phi(t) A(t), \Phi\left(t_{1}\right)=I_{n}(t \in T)$ and $z$ is the solution of $\dot{z}(t)=A(t) z(t)+$ $B(t) w(t), z\left(t_{0}\right)=0$.

Under assumptions (A1) and (A2) the main result in [29, Lemmas 5.1 and 5.2, Theorem 5.1], relating this set to those of [17] and [33], and the second order condition, is the following.

6.7 Theorem: Let $(x, u, p) \in Z \times X$. Then the following holds:

a. $\mathcal{G}_{1}(x, u, p) \subset \mathcal{G}_{2}(x, u, p)$.

b. If $(x, u, p) \in \mathcal{E} \cap \mathcal{L}^{\prime}$ with $(x, u) \in \mathcal{N}\left[a, t_{1}\right]$ for all $a \in\left[t_{0}, t_{1}\right)$, then $\mathcal{C}_{0}(x, u, p) \cap\left(t_{0}, t_{1}\right) \subset \mathcal{G}_{2}(x, u, p)$.

c. If $(x, u, p) \in \mathcal{E}$ and $(x, u)$ is normal to $\mathrm{P}(\mathcal{A})$, then $(x, u, p) \in \mathcal{H} \Rightarrow$ $\mathcal{G}_{2}(x, u, p) \cap\left(t_{0}, t_{1}\right)=\emptyset$.

Note that this set yields a necessary condition for optimality assuming the process under consideration is normal (compare with Theorems 6.2 and 6.4 where normality is not required). In particular, for the linear fixed-endpoint problem, where the dynamics are of the form $\dot{x}(t)=A(t) x(t)+B(t) u(t)$ and second order conditions are easily obtained without normality assumptions, the sets $\mathcal{G}_{1}(x, u, p)$ and $\mathcal{R}_{1}(x, u, p)$ can be applied in the abnormal case but not $\mathcal{G}_{2}(x, u, p)$. A full discussion of this fact can be found in [24].

This is not mentioned in [29]. Instead, two examples are provided for which the results of [17] cannot be applied because both examples lie outside the scope of [17] (one involves a nonconvex control set while, for the other, both endpoints vary). The same occurs with respect to [30] and [33], and the conclusion in [29] is then that the new results are "much sharper."

Now, even for the problem we are considering, the two undesirable fea- 
tures of $\mathcal{G}_{1}(x, u, p)$ explained above remain present in the set defined by Zeidan. Its nonemptiness, in the normal case, implies the existence of a negative second variation, but the converse remains open. Also, verifying membership of this set may be much more difficult than checking if $x \notin \mathcal{H}$. As before, these two features can be solved by the introduction of a new set containing $\mathcal{G}_{2}(x, u, p)$.

6.8 Definition: For any $(x, u, p) \in Z \times X$ let $\mathcal{R}_{2}(x, u, p)$ be the set of points $s \in\left[t_{0}, t_{1}\right)$ for which there exists $(y, v) \in Y_{s}(x, u)$ such that

i. $\int_{s}^{t_{1}}\{\langle y(t), \sigma(t)\rangle+\langle v(t), \rho(t)\rangle\} d t \leq 0$.

ii. If there exists $q \in X_{s}$ such that $\dot{q}(t)+A^{*}(t) q(t)=\sigma(t)$ and $B^{*}(t) q(t)=$ $\rho(t)\left(t \in T_{s}\right)$, then there exists $w:\left[t_{0}, s\right] \rightarrow \mathbf{R}^{m}$ piecewise continuous with $\langle z(s), q(s)\rangle \neq 0$ where $z$ is the solution of $\dot{z}(t)=A(t) z(t)+B(t) w(t), z\left(t_{0}\right)=0$.

6.9 Theorem: Let $(x, u, p) \in Z \times X$. Then the following holds:

a. $\mathcal{R}_{1}(x, u, p) \cup \mathcal{G}_{2}(x, u, p) \subset \mathcal{R}_{2}(x, u, p)$.

b. If $(x, u, p) \in \mathcal{E}$ and $(x, u)$ is normal to $\mathrm{P}(\mathcal{A})$, then $(x, u, p) \in \mathcal{H} \Leftrightarrow$ $\mathcal{R}_{2}(x, u, p)=\emptyset$.

Proof:

(a): Let $s \in \mathcal{R}_{1}(x, u, p)$ and let $(y, v)$ and $(z, w)$ be as in 6.3. Suppose there exists $q \in X_{s}$ such that $\dot{q}(t)+A^{*}(t) q(t)=\sigma(t), B^{*}(t) q(t)=\rho(t)\left(t \in T_{s}\right)$ (if it does not exist then $s \in \mathcal{R}_{2}(x, u, p)$ ). The result clearly follows since $\langle z(s), q(s)\rangle=-\gamma \neq 0$.

Let $s \in \mathcal{G}_{2}(x, u, p)$ and let $(y, v) \in Y_{s}(x, u)$ and $q \in X_{s}$ be as in 6.6. As in the proof of $6.4 \mathrm{a}$, condition 6.8(i) is a consequence of 6.6(ii). Now, suppose there exists $q_{1} \in X_{s}$ such that $\dot{q}_{1}(t)+A^{*}(t) q_{1}(t)=\sigma(t), B^{*}(t) q_{1}(t)=\rho(t)$ $\left(t \in T_{s}\right)$. Note first that

$$
0=\int_{s}^{t_{1}}\{\langle y(t), \sigma(t)\rangle+\langle v(t), \rho(t)\rangle\} d t=-\int_{s}^{t_{1}}\langle v(t), \lambda(t)\rangle d t \leq 0
$$

and so the inequality in 6.6(ii) is equality for all $t \in T_{s}$. Now, let $r \equiv q-q_{1}$ and observe that, since $\dot{r}(t)+A^{*}(t) r(t)=0$ we have $r(t)=\Phi^{*}(t) r\left(t_{1}\right)\left(t \in T_{s}\right)$. Hence $\lambda(t)=B^{*}(t) \Phi^{*}(t) r\left(t_{1}\right)$ and, by 6.6(iii), there exists $w:\left[t_{0}, s\right] \rightarrow \mathbf{R}^{m}$ piecewise continuous such that, if $z$ is the solution of $\dot{z}(t)=A(t) z(t)+B(t) w(t)$, $z\left(t_{0}\right)=0$, then $\left\langle z(s), q_{1}(s)\right\rangle=-\left\langle z(s), \Phi^{*}(s) r\left(t_{1}\right)-q(s)\right\rangle>0$. This shows that $s \in \mathcal{R}_{2}(x, u, p)$.

(b) " $\Rightarrow$ ": Suppose there exists $s \in \mathcal{R}_{2}(x, u, p)$. Let $(y, v) \in Y_{s}(x, u)$ be as in 6.8 and define $(\zeta(t), \eta(t)):=(0,0)$ if $t \in\left[t_{0}, s\right],(\zeta(t), \eta(t)):=(y(t), v(t))$ if $t \in\left(s, t_{1}\right]$. Note that, by $6.8(\mathrm{i})$,

$J_{p}((x, u) ;(\zeta, \eta))=\int_{t_{0}}^{t_{1}} 2 \Omega(t, \zeta(t), \eta(t)) d t=\int_{s}^{t_{1}}\{\langle y(t), \sigma(t)\rangle+\langle v(t), \rho(t)\rangle\} d t \leq 0$.

If the value of the integral in 6.8(i) is negative, we contradict the assumption $(x, u, p) \in \mathcal{H}$. If it vanishes, then $(\zeta, \eta)$ solves the accessory problem (AP) of 
minimizing $J_{p}((x, u), \cdot) / 2$ over $Y(x, u)$. Since $(x, u)$ is normal to $\mathrm{P}(\mathcal{A})$, so is $(\zeta, \eta)$ to $(\mathrm{AP})$. Hence there exists $q_{1} \in X$ such that, for all $t \in T$,

$$
\begin{gathered}
\dot{q}_{1}(t)+A^{*}(t) q_{1}(t)=-H_{x x}(\tilde{x}(t), p(t), 1) \zeta(t)-H_{x u}(\tilde{x}(t), p(t), 1) \eta(t) \\
B^{*}(t) q_{1}(t)=-H_{u x}(\tilde{x}(t), p(t), 1) \zeta(t)-H_{u u}(\tilde{x}(t), p(t), 1) \eta(t) .
\end{gathered}
$$

Let $q$ be the restriction of $q_{1}$ to $\left[s, t_{1}\right]$. By $6.8(\mathrm{ii})$, there exists $w:\left[t_{0}, s\right] \rightarrow \mathbf{R}^{m}$ piecewise continuous with $\langle z(s), q(s)\rangle \neq 0$ where $z$ is the solution of $\dot{z}(t)=$ $A(t) z(t)+B(t) w(t), z\left(t_{0}\right)=0$. This implies, in particular, that $s>t_{0}$. Now, since $\dot{q}_{1}(t)+A^{*}(t) q_{1}(t)=0\left(t \in\left[t_{0}, s\right]\right)$, we have $q_{1}(s)=\Phi^{*}(s) \Phi^{*-1}(t) q_{1}(t)$ $\left(t \in\left[t_{0}, s\right]\right)$, and so

$0 \neq\langle z(s), q(s)\rangle=\int_{t_{0}}^{s}\left\langle\Phi^{-1}(s) \Phi(\tau) B(\tau) w(\tau), q_{1}(s)\right\rangle d \tau=\int_{t_{0}}^{s}\left\langle w(\tau), B^{*}(\tau) q_{1}(\tau)\right\rangle d \tau$.

But $B^{*}(t) q_{1}(t)=0$ for all $t \in\left[t_{0}, s\right]$ and we reach a contradiction.

(b) " $\Leftarrow$ ": Suppose $(x, u, p) \notin \mathcal{H}$. Let $(y, v) \in Y(x, u)$ be such that $J_{p}((x, u) ;(y, v))<0$. Clearly, condition 6.8(ii) does not apply since, otherwise, the integral in (i) would vanish. Thus $t_{0} \in \mathcal{R}_{2}(x, u, p)$.

6.10 Remark: It should be noted that, just like with $\mathcal{G}_{1}(x, u, p)$ (see Remark 6.5), Theorem 6.9 implies that $(x, u, p) \in \mathcal{H} \Rightarrow \mathcal{G}_{2}(x, u, p)=\emptyset$. Therefore, a necessary condition for optimality is the emptiness of $\mathcal{G}_{2}(x, u, p)$ in $\left[t_{0}, t_{1}\right)$. We mention this fact because in [29], as well as in [17], it is emphasized that this necessary condition holds for points in the interior of the time interval. This assertion may be rather misleading since, as it is well-known, Jacobi's necessary condition (in the classical context) only applies for points in the interior of the time interval. On the other hand, Jacobi's strengthened condition states that there are no conjugate points in the half-open interval, and this condition leads to sufficiency (see Theorems 2.2 and 2.10). With the assertion of [17] and [29] one is thus tempted to think that a similar result might hold for the sets $\mathcal{G}_{1}(x, u, p)$ or $\mathcal{G}_{2}(x, u, p)$.

The behaviour of these sets of points is in fact quite different to that of classical conjugate points. For the calculus of variations problem it is wellknown that, if $x \in \mathcal{L}^{\prime} \cap C^{1}$, and $y$ and $q$ of class $C^{1}$ are nonidentically vanishing solutions of the system $(\mathrm{J})$ (see 3.1) then the zeros of $y$ are isolated and, moreover, between any two zeros of $y$ on $T$ there is a zero of $q$ (see [22, p 222]). Each of the sets we are now dealing with, as one readily verifies, is an interval contained in $\left[t_{0}, t_{1}\right)$ (see $\left.[23]\right)$.

Now, the simplicity of the sets $\mathcal{R}_{1}$ and $\mathcal{R}_{2}$, compared with the complexity of $\mathcal{G}_{1}$ and $\mathcal{G}_{2}$, is obvious. Let us end this section with two simple examples which help to illustrate the applicability of these four sets. 
6.11 Example: Consider the problem of minimizing $I(x, u)=\int_{0}^{1} x(t) u(t) d t$ subject to $\dot{x}(t)=x(t)+u(t)(t \in[0,1])$ and $x(0)=x(1)=0$.

In this case, $T=[0,1], n=m=1, \xi_{0}=\xi_{1}=0, \mathcal{A}=T \times \mathbf{R}^{2}$, $L(t, x, u)=x u$ and $f(t, x, u)=x+u$. We have $H(t, x, u, p, 1)=(x+u) p-x u$.

Note first that $2 \Omega(t, y, v)=2 y v$ and so, for any nonzero $(y, v) \in Y(x, u)$ $\left(\dot{y}(t)=y(t)+v(t)\right.$ and $\left.y\left(t_{0}\right)=y\left(t_{1}\right)=0\right)$, we have $J_{p}((x, u) ;(y, v))=$ $-4 \int_{0}^{1} y^{2}(t) d t<0$ implying that $(x, u, p) \notin \mathcal{H}$. Since any process is normal to $\mathrm{P}(\mathcal{A})$, Theorem 4.1 implies that this problem has no solution. Though this conclusion follows trivially, without any use of conjugate points, it is interesting to see how the theory developed so far can or cannot be applied.

First of all, since any process is singular, we cannot apply Theorems 5.8 or 5.10. We also rule out Theorems 5.12 and 5.14 since both $\mathcal{P}(x, u, p)$ and $\mathcal{Q}(x, u, p)$ are empty. Indeed, both require a nonzero $(y, v, q) \in \mathcal{E}_{s}(x, u, p)$, but this set is given by those $(y, v, q) \in Z_{s} \times X_{s}$ satisfying

$$
\dot{y}(t)=y(t)+v(t), \quad \dot{q}(t)+q(t)=v(t), \quad q(t)=y(t) \quad\left(t \in\left[s, t_{1}\right]\right)
$$

and so $\mathcal{E}_{s}(x, u, p)=\{(0,0,0)\}$.

Since the sets of conjugate intervals allow us to consider functions which do not satisfy Jacobi's system, let us try to apply the results of this section. The two sets $\mathcal{R}_{1}$ and $\mathcal{R}_{2}$ are obviously nonempty since, having $\sigma(t)=v(t)$ and $\rho(t)=y(t)$, the integral in the first condition of their definition corresponds to $-2 \int_{s}^{1} y^{2}(t) d t$ for all $(y, v) \in Y_{s}(x, u)$.

Now, for the sets $\mathcal{G}_{1}$ and $\mathcal{G}_{2}$, note first that we require, in particular, the existence of a nonzero $(y, v, q) \in Y_{s}(x, u) \times X_{s}$ sastisfying

i. $\dot{q}(t)+q(t)=v(t)(t \in[s, 1])$.

ii. $v(t)(q(t)-y(t)) \geq 0(t \in[s, 1])$.

We pose the question of how such functions can be found. There seems to be no criterion at all except, possibly, to find a solution of Jacobi's system, yielding an equality in (ii). But, as we already know, this criterion fails in this example. Let us then try with the following functions which first come to mind:

$$
y(t)=\left\{\begin{array}{ll}
t & \text { if } t \in[0,1 / 2] \\
1-t & \text { if } t \in[1 / 2,1],
\end{array} \quad v(t)= \begin{cases}1-t & \text { if } t \in[0,1 / 2] \\
t-2 & \text { if } t \in[1 / 2,1]\end{cases}\right.
$$

In view of (i), we have

$$
q(t)=e^{-t}\left(c+\int_{0}^{t} e^{\tau} v(\tau) d \tau\right)
$$

and so, after evaluating the corresponding integrals, we get

$$
q(t)=e^{-t}\left(c+\int_{0}^{t} e^{\tau}(1-\tau) d \tau\right)=e^{-t}(c-2)-t+2 \quad(t \in[0,1 / 2])
$$




$$
\begin{gathered}
q(t)=e^{-t}\left(c+\int_{0}^{1 / 2} e^{\tau}(1-\tau) d \tau+\int_{1 / 2}^{t} e^{\tau}(\tau-2) d \tau\right) \\
=e^{-t}(c-2)+4 e^{1 / 2-t}+t-3 \quad(t \in[1 / 2,1]) .
\end{gathered}
$$

For (ii) to hold, we need in particular that

$$
\begin{gathered}
0 \leq v(1 / 2-)(q(1 / 2)-y(1 / 2))=\left(e^{-1 / 2}(c-2)+1\right) / 2, \\
0 \leq v(1 / 2+)(q(1 / 2)-y(1 / 2))=-3\left(e^{-1 / 2}(c-2)+1\right) / 2
\end{gathered}
$$

which holds (only) if $c=2-e^{1 / 2}$. One should then verify that, with this constant, condition (ii) holds, namely,

$$
\begin{gathered}
(1-t)\left(e^{-t}(c-2)-2 t+2\right) \geq 0 \quad(t \in[0,1 / 2]) \\
(t-2)\left(e^{-t}(c-2)+4 e^{1 / 2-t}+2 t-4\right) \geq 0 \quad(t \in[1 / 2,1]) .
\end{gathered}
$$

The idea of analyzing this example as far as this point has been to exhibit (as Ewing says), even for such a trivial example, some of the "gymnastics" that in the use of the sets defined by Loewen and Zheng, and Zeidan, one must be prepared to employ.

6.12 Example: Consider the problem of minimizing

$$
I(x, u)=\frac{1}{2} \int_{0}^{\pi}\left\{u^{2}(t) \sin ^{2} t-x^{2}(t)(1+\sin 2 t)\right\} d t
$$

subject to $\dot{x}(t)=x(t)+u(t)(t \in[0, \pi])$ and $x(0)=x(\pi)=0$.

In this case $T=[0,1], n=m=1, \xi_{0}=\xi_{1}=0, \mathcal{A}=T \times \mathbf{R}^{2}, L(t, x, u)=$ $\left[u^{2} \sin ^{2} t-x^{2}(1+\sin 2 t)\right] / 2$ and $f(t, x, u)=x+u$. We have $H(t, x, u, p, 1)=$ $(x+u) p-\left[u^{2} \sin ^{2} t-x^{2}(1+\sin 2 t)\right] / 2$.

Since $H_{u u}(t, x, u, p, 1)=-\sin ^{2} t$, any process is singular and Theorems 5.8 and 5.10 cannot be applied. We could try to apply Theorems 5.12 or 5.14 but we face a problem. We need to find a nonzero $(y, v, q) \in \mathcal{E}_{s}(x, u, p)$ with $y(s)=y\left(t_{1}\right)=0$. The set $\mathcal{E}_{s}(x, u, p)$ is given by those $(y, v, q) \in Z_{s} \times X_{s}$ satisfying

$\dot{y}(t)=y(t)+v(t), \quad \dot{q}(t)+q(t)=-y(t)(1+\sin 2 t), \quad q(t)=v(t) \sin ^{2} t \quad\left(t \in\left[s, t_{1}\right]\right)$.

As one readily verifies, if $y$ satisfies the differential equation

$$
\ddot{y}(t)+2 \dot{y}(t) \cot t+y(t) \cot ^{2} t=0
$$

the above relations hold. However, the solution of this equation, given in [21, equation 2.1.6.55], is expressed in terms of the Gauss hypergeometric equation, 
and we prefer not even to copy it. As we shall see below, with the sets $\mathcal{R}_{1}$ and $\mathcal{R}_{2}$, there is no need to deal with such an equation at all.

Let us prove this claim. First, we have $\sigma(t)=-y(t)(1+\sin 2 t)$ and $\rho(t)=v(t) \sin ^{2} t$, and so the integral in the definition of $\mathcal{R}_{1}$ and $\mathcal{R}_{2}$ is given by

$$
\int_{s}^{\pi}\left\{v^{2}(t) \sin ^{2} t-y^{2}(t)(1+\sin 2 t)\right\} d t .
$$

Setting $(y(t), v(t)):=(\sin t, \cos t-\sin t)(t \in[0, \pi])$, this integral, as one readily verifies, vanishes for $s=0$. Thus $(y, v) \in Y_{s}(x, u)$ with $s=0$ is such that $6.3(\mathrm{i})$ and 6.8(i) hold. The requirement of the second condition in the definition of $\mathcal{R}_{2}$ must be met if there exists $q \in X_{s}$ such that

$\dot{q}(t)+q(t)=-\sin t(1+\sin 2 t) \quad$ and $\quad q(t)=\cos t \sin ^{2} t-\sin ^{3} t \quad(t \in[0, \pi])$.

But both relations are incompatible and so $s=0$ belongs to this set. Since any process is normal to $\mathrm{P}(\mathcal{A})$, Theorem 6.9 implies that this problem has no solution. Note that $\mathcal{R}_{2}$ has the advantage over $\mathcal{R}_{1}$ that we do not have to check the existence of $(z, w) \in Y(x, u)$ satisfying 6.3(ii). Also, let us mention that the choice of $(y, v)$ above is not an idle one. For a wide range of problems (like this example) one can establish simple criteria for finding $(y, v) \in Y_{s}(x, u)$ for which the integral in 6.8(i) vanishes, but the corresponding condition (ii) is immaterial (see [4]).

Let us now proceed with the sets $\mathcal{G}_{1}$ and $\mathcal{G}_{2}$. In both cases we require, in particular, the existence of a nonzero $(y, v, q)$ in $Y_{s}(x, u) \times X_{s}$ such that

i. $\dot{q}(t)+q(t)=-y(t)(1+\sin 2 t)(t \in[s, \pi])$.

ii. $v(t)\left(q(t)-v(t) \sin ^{2} t\right) \geq 0(t \in[s, \pi])$.

As in the previous (and any other) example, we come to the question of how to prove the existence of such functions. Again, the only possible criterion seems to be that of finding a solution of Jacobi's system (contrary to what happens, as just explained, with $\mathcal{R}_{1}$ and $\mathcal{R}_{2}$ ), but this seems to be extremely complicated for this example. Let us then try with the functions used to show that $\mathcal{R}_{2}(x, u, p)$ is nonempty.

We have $(y(t), v(t))=(\sin t, \cos t-\sin t)(t \in[0, \pi])$, and $s=0$. Observe first that (i) holds if and only if there exists $c \in \mathbf{R}$ such that

$$
q(t)=e^{-t}\left(c-\int_{0}^{t} e^{\tau} y(\tau)(1+\sin 2 \tau) d \tau\right) .
$$

After a long series of calculations and integrations by parts, we finally arrive at the following expression:

$q(t)=e^{-t}\left(c-\frac{1}{2}\left[e^{t}(\sin t-\cos t)+1\right]-\frac{1}{5}\left[2 e^{t} \sin ^{2} t(\sin t+\cos t)-e^{t} \cos ^{2} t(\sin t-\cos t)-1\right]\right)$. 
Condition (ii) requires that

$$
h(t):=(\cos t-\sin t)\left[q(t)-(\cos t-\sin t) \sin ^{2} t\right] \geq 0 \quad \text { for all } t \in[0, \pi] .
$$

In particular, we have $0 \leq h(0)=q(0)=c$. Also, for example,

$$
h(\pi / 2)=-q(\pi / 2)-1=-e^{-\pi / 2}\left(c-\frac{3}{10}\right)-\frac{1}{10} \geq 0 .
$$

But this relation holds only if $c \leq\left(3-e^{\pi / 2}\right) / 10<0$. In other words, if $(y(t), v(t))=(\sin t, \cos t-\sin t)(t \in[0, \pi])$, we reach a contradiction in the definition of $\mathcal{G}_{1}$ and $\mathcal{G}_{2}$. We prefer to proceed no further with the task of trying to prove (the uncertain) nonemptiness of these sets.

\section{A new set}

From Theorems 6.4 and 6.9 we know that $\mathcal{G}_{1}(x, u, p) \subset \mathcal{R}_{1}(x, u, p) \cup$ $\mathcal{G}_{2}(x, u, p) \subset \mathcal{R}_{2}(x, u, p)$. Moreover, $(x, u, p) \in \mathcal{H} \Leftrightarrow \mathcal{R}_{1}(x, u, p)=\emptyset$ and, in the normal case, $(x, u, p) \in \mathcal{H} \Leftrightarrow \mathcal{R}_{2}(x, u, p)=\emptyset$. Also, the first approach to conjugacy given in Section 5 is related to $\mathcal{R}_{2}$ since, under the assumptions of Theorem 5.10a, $\mathcal{C}_{0}(x, u, p) \cap\left(t_{0}, t_{1}\right) \subset \mathcal{R}_{2}(x, u, p)$. Finally, the conditions defining membership of $\mathcal{R}_{1}$ and $\mathcal{R}_{2}$ are of a much simpler nature than those for $\mathcal{G}_{1}$ and $\mathcal{G}_{2}$.

In view of these facts, $\mathcal{R}_{2}$ (and, for the abnormal case, also $\mathcal{R}_{1}$ ) seems to solve various disadvantages inherent in other sets previously defined. However, nothing has been said with respect to the sets $\mathcal{P}$ and $\mathcal{Q}$ defined in Section 5 . The set we now introduce unifies the two approaches explained in that section. As one readily verifies it contains, for points in the open time interval, both $\mathcal{P}$ and $\mathcal{Q}$.

7.1 Definition: For any $(x, u, p) \in Z \times X$ let $\mathcal{S}(x, u, p)$ be the set of points $s \in\left[t_{0}, t_{1}\right)$ for which there exists $(y, v) \in Y_{s}(x, u)$ such that

i. $\int_{s}^{t_{1}}\{\langle y(t), \sigma(t)\rangle+\langle v(t), \rho(t)\rangle\} d t \leq 0$.

ii. If there exists $q \in X_{s}$ such that $\dot{q}(t)+A^{*}(t) q(t)=\sigma(t)$ and $B^{*}(t) q(t)=$ $\rho(t)\left(t \in T_{s}\right)$, then $s>t_{0}$ and either (a) or (b) holds:

a. There exists $\epsilon>0$ such that $(x, u) \in \mathcal{N}([s-\epsilon, s] \cap T), y \not \equiv 0$ on $[s, s+\epsilon] \cap T$, and $H_{u u}(\tilde{x}(t), p(t), 1)<0$ for all $t \in[s-\epsilon, s+\epsilon] \cap T$.

b. $H_{u u}(\tilde{x}(s), p(s), 1) v(s) \neq 0$ and $B$ is continuous at $s$.

7.2 Theorem: If $(x, u, p) \in \mathcal{E}$ with $(x, u)$ normal to $\mathrm{P}(\mathcal{A})$, then $(x, u, p) \in$ $\mathcal{H} \Leftrightarrow \mathcal{S}(x, u, p)=\emptyset$.

Proof:

" $\Rightarrow$ ": Suppose there exists $s \in \mathcal{S}(x, u, p)$. Let $(y, v) \in Y_{s}(x, u)$ be as in 7.1 and proceed exactly as in the proof of $6.9 \mathrm{~b}$ up to "Let $q$ be the restriction 
of $q_{1}$ to $\left[s, t_{1}\right]$." The existence of this function implies that $s>t_{0}$. Now, if (a) (resp (b)) holds, we proceed as in the proof of Theorem 5.12 (resp 5.14) replacing $(z, w, r)$ with $\left(\zeta, \eta, q_{1}\right)$.

"६": Analogous to the proof of $6.9(\mathrm{~b})$.

Note that, in the examples of the previous section, all the statements concerning $\mathcal{R}_{2}$ remain valid for $\mathcal{S}$. These two sets can be related to each other if one imposes certain conditions on $B$ and/or normality on subintervals. For the case when $\mathcal{S}$ is expressed only in terms of condition (b), we refer to [25] for an account of such relations.

Let us end this "conjugate journey" by showing that, in general, conditions (a) and (b) in Definition 7.1 are not equivalent. In the two examples that follow we show that a certain point belongs to $\mathcal{S}(x, u, p)$, and this follows by an application of one, and only one, of these two conditions.

7.3 Example: Consider the problem of Example 5.15 where $H(t, x, u, p, 1)=$ $p \varphi(t) u-\frac{1}{2}\left(u^{2}-x^{2}\right)$ and

$$
\varphi(t)= \begin{cases}0 & \text { if } t \in[-\pi,-1] \\ h(t) & \text { if } t \in[-1,0] \\ 1 & \text { if } t \in[0, \pi]\end{cases}
$$

with $h:[-1,0] \rightarrow \mathbf{R}$ any positive, continuous function.

Let $(x, u, p) \in Z \times X$. We have $\sigma(t)=-y(t)$ and $\rho(t)=v(t)$ and, so, if we set $(y(t), v(t)):=(\sin t, \cos t)$ for all $t \in[0, \pi]$, the integral in 7.1(i) vanishes for $s=0$. Setting $q(t):=\cos t(t \in[0, \pi])$, also 7.1(ii) is satisfied. Since $s>-\pi$ and (a) holds with, for example, $\epsilon=\pi$, we conclude that $0 \in S(x, u, p)$. Note that, for $\mathcal{R}_{2}(x, u, p)$, we can proceed similarly but we still need to prove (which, for this example, is straightforward) the existence of $w:[-\pi, 0] \rightarrow \mathbf{R}$ piecewise continuous with $z(0) q(0)=z(0) \neq 0$ where $z$ is the solution of $\dot{z}(t)=\varphi(t) w(t)$, $z(-\pi)=0$. Now, clearly, condition (b) fails unless $\varphi$ is continuous at 0 , which occurs only if $h(0)=1$. Thus, if $h(0) \neq 1,(\mathrm{a}) \nRightarrow(\mathrm{b})$.

7.4 Example: Consider the problem of minimizing

$$
I(x, u)=\frac{1}{2} \int_{-\pi}^{\pi} \varphi(t)\left\{u^{2}(t)-x^{2}(t)\right\} d t
$$

subject to $\dot{x}(t)=u(t)(t \in[-\pi, \pi])$ and $x(-\pi)=x(\pi)=0$, where $\varphi(t)=0$ $(t \in[-\pi, 0)), \varphi(t)=1(t \in[0, \pi])$.

We have $H(t, x, u, p, 1)=p u-\frac{1}{2} \varphi(t)\left\{u^{2}-x^{2}\right\}$. Let $(x, u, p) \in Z \times X$ and note that $\sigma(t)=-\varphi(t) y(t)$ and $\rho(t)=\varphi(t) v(t)$. If, as in the other example, we set $(y(t), v(t)):=(\sin t, \cos t)$ for all $t \in[0, \pi]$, the integral in 7.1(i) vanishes for $s=0$. Setting $q(t):=\cos t(t \in[0, \pi])$, also 7.1 (ii) is satisfied. We have $s>-\pi$ 
and (b) holds since $H_{u u}(\tilde{x}(0+), p(0+), 1) v(0)=-\varphi(0+) \neq 0$ and $B \equiv 1$ is continuous at $s=0$. Therefore $0 \in \mathcal{S}(x, u, p)$. On the other hand, since $H_{u u}(\tilde{x}(t), p(t), 1)=-\varphi(t)$, there is no $\epsilon>0$ for which $H_{u u}(\tilde{x}(t), p(t), 1)<0$ for all $t \in[-\epsilon, \epsilon]$. Thus $(\mathrm{b}) \not \Rightarrow(\mathrm{a})$.

\section{References}

[1] Alekseev VM, Tikhomirov VM, Fomin SV (1987) Optimal Control, Consultants Bureau, New York

[2] Berlanga R, Rosenblueth JF (2002) Jacobi's condition for singular extremals: an extended notion of conjugate points, Applied Mathematics Letters, 15: 453-458

[3] Berlanga R, Rosenblueth JF (2004) A Sturm-Liouville approach applicable to different notions of conjugacy, Applied Mathematics Letters, 17: $467-472$

[4] Berlanga R, Rosenblueth JF (2004) Extended conjugate points in the calculus of variations, IMA Journal of Mathematical Control \& Information, 21: 159-173

[5] Bernhard P (1983) La théorie de la seconde variation et le problème linéaire quadratique, Advances in Hamiltonian systems (Rome 1981), Birkhäuser Boston, 109-142

[6] Bliss GA (1946) Lectures on the Calculus of Variations, University of Chicago Press, Chicago

[7] Bowman F (1958) Introduction to Bessel Functions, Dover Publications, New York

[8] Breakwell JV, Ho YC (1965) On the conjugate point condition for the control problem, International Journal of Engineering Science, 2: 565-579

[9] Brechtken-Manderscheid U (1983) Introduction to the Calculus of Variations, English edition 1991, Chapman \& Hall, London

[10] Ewing GM (1985) Calculus of Variations with Applications, Dover Publications, New York

[11] Fleming WH, Rishel RW (1975) Deterministic and Stochastic Optimal Control, Springer-Verlag, New York

[12] Gelfand IM, Fomin SV (1963) Calculus of Variations, Prentice-Hall, New Jersey

[13] Gilbert EG, Bernstein DS (1983) Second-order necessary conditions in optimal control: Accessory-problem results without normality conditions, Journal of Optimization Theory \& Applications, 41: 75-106

[14] Hestenes MR (1966) Calculus of Variations and Optimal Control Theory, John Wiley \& Sons, New York

[15] Leitmann G (1981) The Calculus of Variations and Optimal Control, Plenum Press, New York

[16] Loewen PD (1990) Second-order sufficiency criteria and local convexity for equivalent problems in the calculus of variations, Journal of Mathematical Analysis \& Applications, 146: 512-522 
[17] Loewen PD, Zheng H (1994) Generalized conjugate points for optimal control problems, Nonlinear Analysis, Theory, Methods \& Applications, 22: $771-791$

[18] Morse M (1973) Variational Analysis: Critical Extremals and Sturmian Extensions, John Wiley \& Sons, New York

[19] Neustadt LW (1976) Optimization, A Theory of Necessary Conditions, Princeton University Press, Princeton

[20] Osmolovskii NP (1975) Second-order conditions for a weak local minimum in an optimal control problem (necessity, sufficiency), Soviet Math Dokl, 16: 1480-1484

[21] Polyanin AD, Zaitsev VF (1995) Handbook of Exact Solutions for Ordinary Differential Equations, CRC Press, Boca Raton, Florida

[22] Reid WT (1971) Ordinary Differential Equations, John Wiley \& Sons, New York

[23] Rosenblueth JF (2002) Conjugate intervals for singular trajectories in the calculus of variations, Memorias del XXXIV Congreso Nacional de la Sociedad Matemática Mexicana, Aportaciones Matemáticas, Serie Comunicaciones 30: 81-98

[24] Rosenblueth JF (2003) Conjugate intervals for the linear fixed-endpoint control problem, Journal of Optimization Theory \& Applications, 116: 393-420

[25] Sánchez Licea G (2003) Reforzamientos de la Condición de Weierstrass y Conjugación en Control Optimo, IIMAS-UNAM, PhD Thesis

[26] Wan FYM (1995) Introduction to the Calculus of Variations and its Applications, Chapman \& Hall, New York

[27] Warga J (1978) A second-order Lagrangian condition for restricted control problems, Journal of Optimization Theory \& Applications, 24: $475-483$

[28] Zeidan V (1994) The Riccati equation for optimal control problems with mixed state-control constraints: necessity and sufficiency, SIAM Journal on Control \& Optimization, 32: 1297-1321

[29] Zeidan V (1996) Admissible directions and generalized coupled points for optimal control problems, Nonlinear Analysis, Theory, Methods \& Applications, 26: 479-507

[30] Zeidan V, Zezza P (1988) The conjugate point condition for smooth control sets, Journal of Mathematical Analysis \& Applications, 132: 572589

[31] Zeidan V, Zezza P (1988) Necessary conditions for optimal control problems: conjugate points, SIAM Journal on Control \& Optimization, 26: $592-608$

[32] Zeidan V, Zezza P (1989) Conjugate points and optimal control: counterexamples, IEEE Transactions on Automatic Control, 34: 254-255

[33] Zeidan V, Zezza P (1991) Coupled points in optimal control theory, IEEE Transactions on Automatic Control, 36: 1276-1281

Received: April 20, 2006 